\title{
Heavy-hadron molecular spectrum from light-meson exchange saturation
}

\author{
Fang-Zheng Peng $\odot,{ }^{1}$ Mario Sánchez Sánchez $\odot,{ }^{2}$ Mao-Jun Yan $\odot,{ }^{1}$ and Manuel Pavon Valderrama $\odot^{1, *}$ \\ ${ }^{1}$ School of Physics, Beihang University, Beijing 100191, China \\ ${ }^{2}$ Centre d'Études Nucléaires, CNRS/IN2P3, Université de Bordeaux, 33175 Gradignan, France
}

(Received 27 February 2021; accepted 10 December 2021; published 25 February 2022)

\begin{abstract}
If known, the spectrum of heavy-hadron molecules will be a key tool to disentangle the nature of the exotic states that are being discovered in experiments. Here we argue that the general features of the molecular spectrum can be deduced from the idea that the short-range interaction between the heavy hadrons is effectively described by scalar- and vector-meson exchange, i.e., the $\sigma, \rho$, and $\omega$ mesons. By means of a contact-range theory, where the couplings are saturated by the aforementioned light mesons, we are indeed able to postdict the $X(3872)$ (as a $D^{*} \bar{D}$ molecule) from the three $P_{c}(4312), P_{c}(4440)$, and $P_{c}(4457)$ pentaquarks (as $\bar{D} \Sigma_{c}$ and $\bar{D}^{*} \Sigma_{c}$ molecules). We predict a $J^{P C}=1^{--} D \bar{D}_{1}$ molecule at $4240-4260 \mathrm{MeV}$, which might support the hypothesis that the $Y(4260)$ is at least partly molecular. The extension of these ideas to the light baryons requires minor modifications, after which we recover approximate SU(4)-Wigner symmetry in the two-nucleon system and approximately reproduce the masses of the deuteron and the virtual state.
\end{abstract}

DOI: 10.1103/PhysRevD.105.034028

\section{INTRODUCTION}

Theoretical predictions of the hadronic spectrum are fundamental for testing our understanding of strong interactions against experiments. SU(3)-flavor symmetry $[1,2]$, the quark model [3-6], and the theory behind quarkonium [7-10] have provided valuable insights and clear predictions about which hadrons to expect and their approximate masses. With the experimental observation of exotic hadrons-hadrons that are neither three-quark states, a quark-antiquark pair, or that do not fit into preexisting quark-model predictions [11-14]—new theoretical explanations have appeared, among which molecular hadrons $[15,16]$ are popular. However, the spectrum of molecular hadrons is not properly understood, with most theoretical applications of this idea being explicitly customized to explain a particular hadron or a few at most.

The present manuscript attempts to overcome this limitation by proposing a more general explanation of the spectrum of hadronic molecules (in the spirit of Refs. [17,18]). The idea is as follows: first, we will describe the interaction between two heavy hadrons in terms of a contact-range potential. If the range of the binding

\footnotetext{
*mpavon@buaa.edu.cn
}

Published by the American Physical Society under the terms of the Creative Commons Attribution 4.0 International license. Further distribution of this work must maintain attribution to the author(s) and the published article's title, journal citation, and DOI. Funded by SCOAP. mechanism between two heavy hadrons is indeed shorter than the size of the molecular state formed by the aforementioned hadrons, then a contact-range theory will represent a good description. Second, we will assume that the couplings in the contact-range potential are saturated by light-meson exchange (i.e., $\sigma, \rho$, and $\omega$ ) within the saturation procedure of Ref. [19]. Third, for effectively combining the contribution from the saturation of scalarand vector-meson exchange, which happen at a different renormalization scale as the masses of these light mesons are different, we will follow a renormalization group equation (RGE). This RGE will tell us what is the importance of scalar- and vector-meson contributions to saturation relative to each other. Fourth, the couplings derived from saturation are expected to be valid modulo a proportionality constant, which we fix by solving the bound state equation for a molecular candidate, for instance, the $P_{c}(4312)$. Finally, we can derive the predictions of this procedure for other molecular states.

\section{SATURATION}

We will consider a generic two heavy-hadron system $H_{1} H_{2}$, with $H_{i}=D^{(*)}, \Sigma_{c}^{(*)}$, etc. for $i=1,2$. Owing to heavy-quark spin symmetry, their interaction only depends on the light-quark spins of heavy-hadrons $H_{1}$ and $H_{2}$, which we denote $\vec{S}_{L 1}$ and $\vec{S}_{L 2}$. If parity and light spin are conserved in each vertex (i.e., the $\mathrm{H}_{1} \mathrm{H}_{2} \rightarrow \mathrm{H}_{2} \mathrm{H}_{1}$ transition does not happen), then we can describe the $H_{1} H_{2}$ system with a contact-range interaction that admits the multipolar expansion, 


$$
V_{C}=C_{0}+C_{1} \hat{\vec{S}}_{L 1} \cdot \hat{\vec{S}}_{L 2}+\ldots
$$

plus higher order terms, if present, with no momentum/ energy dependence and where $\hat{\vec{S}}_{L i}=\vec{S}_{L i} / S_{L i}$ is a reduced spin operator for hadron $i=1,2$. Higher multipoles can be built analogously, but in practice the monopolar and dipolar terms are more than enough to effectively describe the short-range interaction in most molecules, as higher multipoles will be suppressed [19]. By using a contact theory, we are assuming that pion dynamics and coupled channel effects are perturbative corrections $[20,21]$ and that the resulting two-body bound state is not compact enough as to resolve the short-distance details of the light-meson exchanges binding it. Finally, this contact-range potential has to be regularized, for which a regulator function depending on a cutoff $\Lambda$ is introduced and where the couplings become functions of this cutoff, i.e., $C_{J}=C_{J}(\Lambda)$ for $J=0,1$. Concrete regularization details will be discussed later.

To determine the $C_{0}$ and $C_{1}$ couplings, we assume that they are saturated with scalar- and vector-meson exchange. We begin by writing the Lagrangians for light-meson exchange in a suitable notation in which, instead of the full heavy-hadron fields, we use effective nonrelativistic fields with the quantum numbers of the light quarks within the hadrons [19,22]. This is motivated by the observation that in heavy-hadron interactions the heavy quarks are spectators. For the interaction of a scalar meson with the light-quark degrees of freedom inside a heavy hadron, the Lagrangian reads

$$
\mathcal{L}=g_{S} q_{L}^{\dagger} \sigma q_{L},
$$

where $g_{S}$ is a coupling constant, $\sigma$ the scalar meson field, and $q_{L}$ the aforementioned effective nonrelativistic "lightquark subfield." For the vector mesons, the Lagrangian can be written as a multipole expansion,

$$
\begin{aligned}
\mathcal{L} & =\mathcal{L}_{E 0}+\mathcal{L}_{M 1}+\ldots \\
& =g_{V} q_{L}^{\dagger} V_{0} q_{L}+\frac{f_{V}}{2 M} q_{L}^{\dagger} \epsilon_{i j k} \hat{\vec{S}}_{L i} \partial_{j} V_{k} q_{L}+\ldots,
\end{aligned}
$$

where the dots indicate higher order multipoles. In this Lagrangian, $g_{V}$ and $f_{V}$ are coupling constants, $\epsilon_{i j k}$ the LeviCivita symbol, $V_{\mu}=\left(V_{0}, \vec{V}\right)$ is the vector-meson field, and $M$ is the characteristic mass scale for this multipolar expansion, which for convenience we will set to be the nucleon mass, $M=m_{N} \simeq 938.9 \mathrm{MeV}$. For simplicity, we are not writing down explicitly the isospin or flavor indices. The number of terms depends on the spin of the light-quark degrees of freedom, where for $S_{L}=0\left(\Lambda_{c}\right)$ there is only the electric term, for $S_{L}=\frac{1}{2}\left(D^{(*)}\right)$ there is also the magnetic dipole term, for $S_{L}=1\left(\Sigma_{c}^{(*)}\right)$ an electric quadrupole term (which we will ignore), and so on.
In the $H_{1} H_{2}$ system the potential for the scalar meson is

$$
V_{S}(\vec{q})=-\frac{g_{S 1} g_{S 2}}{\vec{q}^{2}+m_{S}^{2}},
$$

with $\vec{q}$ the exchanged momentum and where $g_{S i}$ refers to the scalar coupling for hadron $i=1,2$, while for the vector mesons we have

$$
\begin{gathered}
V_{E 0}(\vec{q})=+\frac{g_{V 1} g_{V 2}}{\vec{q}^{2}+m_{V}^{2}} \\
V_{M 1}(\vec{q})=+\frac{2}{3} \frac{f_{V 1} f_{V 2}}{4 M^{2}} \hat{\vec{S}}_{L 1} \cdot \hat{\vec{S}}_{L 2} \frac{m_{V}^{2}}{\vec{q}^{2}+m_{V}^{2}}+\ldots
\end{gathered}
$$

with $g_{V i}, f_{V i}$ the couplings for hadron $i=1,2$ and where the dots indicate terms that vanish for $S$-wave or contactrange terms, for which the range is shorter than vectormeson exchange. Following Ref. [19], the saturation condition for scalar-meson exchange reads

$$
C_{0}^{S}\left(\Lambda \sim m_{S}\right) \propto-\frac{g_{S 1} g_{S 2}}{m_{S}^{2}},
$$

where we stress that saturation is expected to work for $\Lambda$ of the same order of magnitude as the mass of the exchanged light meson (thus $\Lambda \sim m_{S}$ ). For vector-meson exchange, we have

$$
\begin{aligned}
& C_{0}^{V}\left(\Lambda \sim m_{V}\right) \propto \frac{g_{V 1} g_{V 2}}{m_{V}^{2}}\left[\zeta+\hat{\vec{I}}_{1} \cdot \hat{\vec{I}}_{2}\right], \\
& C_{1}^{V}\left(\Lambda \sim m_{V}\right) \propto \frac{f_{V 1} f_{V 2}}{6 M^{2}}\left[\zeta+\hat{\vec{I}}_{1} \cdot \hat{\vec{I}}_{2}\right],
\end{aligned}
$$

where we have now included isospin explicitly, $\zeta= \pm 1$ is a sign to indicate the contribution from the omega $(+1$ for $D D, \Sigma_{c} \bar{D}, \Sigma_{c} \Sigma_{c}$ and -1 for $\left.D \bar{D}, \Sigma_{c} D, \Sigma_{c} \bar{\Sigma}_{c}\right)$, and $\hat{\vec{I}}_{i}=\vec{I}_{i} / I_{i}$ are normalized isospin operators for the rho contribution (with $\vec{I}_{i}$ the standard isospin operator and $I_{i}$ the isospin of hadron $i=1,2)$.

\section{RENORMALIZATION GROUP EVOLUTION}

The saturation of the $C_{0}$ coupling receives contributions from two types of light mesons with different masses. To combine the saturation from scalar- and vectormeson exchange into a single coupling, we have to know first the RGE of the couplings, which for nonrelativistic contact-range theories is well understood and follows the equation [23,24]

$$
\frac{d}{d \Lambda}\left[\frac{C(\Lambda)}{\Lambda^{\alpha}}\right]=0 \text { or, } \quad \text { equivalently: } \frac{C\left(\Lambda_{1}\right)}{\Lambda_{1}^{\alpha}}=\frac{C\left(\Lambda_{2}\right)}{\Lambda_{2}^{\alpha}},
$$


with $\alpha$ the anomalous dimension of the coupling. From this we can combine the scalar- and vector-meson contributions as

$$
C^{\mathrm{sat}}\left(m_{V}\right)=C^{V}\left(m_{V}\right)+\left(\frac{m_{V}}{m_{S}}\right)^{\alpha} C^{S}\left(m_{S}\right)
$$

The anomalous dimension is linked with the behavior of the two-body wave function $\Psi(R)$ at distances $R^{-1} \sim \Lambda$ by $|\Psi(R \sim 1 / \Lambda)|^{2} \sim \Lambda^{-\alpha}[23,24]$ (i.e., $\alpha$ encodes the shortrange suppression of the wave function). We do not know the exact form of the short-distance wave function, but owing to the large mass of the heavy hadrons, it is sensible to assume that the semiclassical approximation applies. From including the Langer correction [25], we estimate $\Psi(R) \sim R^{1 / 2}$, which implies $\alpha=+1$. We end up with

$$
\begin{aligned}
C^{\mathrm{sat}}\left(\Lambda=m_{V}\right)= & C_{0}^{\mathrm{sat}}+C_{1}^{\mathrm{sat}} \hat{\vec{S}}_{L 1} \cdot \hat{\vec{S}}_{L 2} \\
\propto & g_{V 1} g_{V 2}\left[\zeta+\hat{T}_{12}\right]\left(\frac{1}{m_{V}^{2}}+\frac{\kappa_{V 1} \kappa_{V 2}}{6 M^{2}} \hat{C}_{L 12}\right) \\
& -\left(\frac{m_{V}}{m_{S}}\right)^{\alpha} \frac{g_{S 1} g_{S 2}}{m_{S}^{2}},
\end{aligned}
$$

where $\hat{T}_{12}=\hat{\vec{I}}_{1} \cdot \hat{\vec{I}}_{2}, \hat{C}_{L 12}=\hat{\vec{S}}_{L 1} \cdot \hat{\vec{S}}_{L 2}$ and $\kappa_{V i}=f_{V i} / g_{V i}$. However, we do not know (yet) the proportionality constant. It is worth stressing that the previous relations implicitly assume that the contact-range interaction has indeed been regularized and that the regularization scale (i.e., the cutoff $\Lambda$ ) is of the right order of magnitude $\left(\Lambda \sim m_{V}\right)$. In the next lines we will explain in more detail our regularization prescription.

\section{PREDICTIONS}

We now explain how to make predictions with the saturated coupling and overcome the ambiguities in its exact definition. First, we regularize the potential

$$
\left\langle p^{\prime}\left|V_{C}\right| p\right\rangle=C_{\mathrm{mol}}^{\mathrm{sat}}\left(\Lambda_{H}\right) f\left(\frac{p^{\prime}}{\Lambda_{H}}\right) f\left(\frac{p}{\Lambda_{H}}\right),
$$

where $C_{\mathrm{mol}}^{\mathrm{sat}}$ is the saturated coupling of Eq. (12) particularized for a given molecule, $f(x)$ is a regulator function (for which we choose a Gaussian regulator, $f(x)=e^{-x^{2}}$ ), and $\Lambda_{H}$ a "physical" cutoff, i.e., a cutoff which corresponds with the natural hadronic momentum scale. The cutoff $\Lambda_{H}$ can be either the scale at which saturation is expected to work (from $m_{S}$ to $m_{V}$ ) or the momentum scale at which we begin to see the internal structure of the heavy hadrons, as these two scales are of the same order of magnitude. We opt for the later: $\Lambda_{H}=1 \mathrm{GeV}$. This contact-range potential can be introduced within a bound state equation to make predictions,

$$
1+2 \mu_{\mathrm{mol}} C_{\mathrm{mol}}^{\mathrm{sat}}\left(\Lambda_{H}\right) \int_{0}^{\infty} \frac{p^{2} d p}{2 \pi^{2}} \frac{f\left(p / \Lambda_{H}\right)^{2}}{p^{2}+\gamma_{\mathrm{mol}}^{2}}=0,
$$

where $\mu_{\mathrm{mol}}$ is the reduced mass of the two-hadron system under consideration and $\gamma_{\text {mol }}$ the wave number of the bound state, related to the binding energy by $B_{\mathrm{mol}}=-\gamma_{\mathrm{mol}}^{2} / 2 \mu_{\mathrm{mol}}$. The mass of the predicted molecular state will be $M_{\text {mol }}=M_{\text {thres }}-B_{\text {mol }}$, with $M_{\text {thres }}=M_{1}+M_{2}$ the threshold of the two-hadron system and $M_{i}$ the mass of hadron $i=1,2$.

The proportionality constant between the contact-range coupling and the saturation ansatz can be determined from a known molecular candidate. Actually, the strength of the interaction is dependent on the reduced mass times the coupling, $\mu_{\mathrm{mol}} C_{\mathrm{mol}}^{\mathrm{sat}}$. If we take the $P_{c}(4312)$ as the reference molecule ${ }^{1}\left(\mathrm{~mol}=P_{c}\right)$, then we can first determine its coupling from solving Eq. (14) for this system, and define the ratio

$$
R_{\mathrm{mol}}=\frac{\mu_{\mathrm{mol}} C_{\mathrm{mol}}^{\mathrm{sat}}}{\mu_{P_{c}} C_{P_{c}}^{\mathrm{sat}}},
$$

from which we can determine the interaction strength of a particular molecule relative to the $P_{c}(4312)$. Finally, we plug $R_{\mathrm{mol}}$ and $C_{P_{c}}^{\text {sat }}$ into Eq. (14),

$$
1+\left(2 \mu_{P_{c}} C_{P_{c}}^{\mathrm{sat}}\right) R_{\mathrm{mol}} \int_{0}^{\infty} \frac{p^{2} d p}{2 \pi^{2}} \frac{f\left(p / \Lambda_{H}\right)^{2}}{p^{2}+\gamma_{\mathrm{mol}}^{2}}=0,
$$

and compute $\gamma_{\mathrm{mol}}$ and $B_{\mathrm{mol}}$ for a particular molecule.

For this we need $g_{V}, \kappa_{V}\left(=f_{V} / g_{V}\right)$, and $g_{S}$ for the heavy hadrons; $g_{V}$ and $\kappa_{V}$ are determined from the mixing of the neutral vector mesons with the electromagnetic current (i.e., Sakurai's universality and vector-meson dominance [26-28]), for which we apply the substitution rules,

$$
\rho_{\mu}^{0} \rightarrow \frac{e}{2 g} A_{\mu} \quad \text { and } \quad \omega_{\mu} \rightarrow \frac{e}{6 g} A_{\mu},
$$

to Eq. (3) and match to the light-quark contribution to the electromagnetic Lagrangian $\left(\rho_{\mu}^{0}\right.$ and $\omega_{\mu}$ are the neutral rho and omega fields, $A_{\mu}$ the photon field, $\mu$ a Lorentz index, $e$ the proton charge, and $g=m_{V} / 2 f_{\pi} \simeq 2.9$ the universal vector-meson coupling constant, with $m_{V}$ the vector-meson mass and $f_{\pi} \simeq 132 \mathrm{MeV}$ the pion weak decay constant). For $g_{V}$ we obtain $g_{V}=g(2 g)$ for $D^{(*)} / D_{0(1)}^{(*)} / D_{1(2)}^{(*)}\left(\Sigma_{c}^{(*)}\right)$; $\kappa_{V}$ is proportional to the (light-quark) magnetic moment of the heavy hadrons, which we calculate in the nonrelativistic quark model [29], yielding $\kappa_{V}=\frac{3}{2}\left(\mu_{u} / \mu_{N}\right)$ for $D^{(*)}$ and $\Sigma_{c}^{(*)}, \kappa_{V}=\frac{3}{2}\left(\mu_{u} / 3 \mu_{N}\right)\left(\frac{3}{2}\left(2 \mu_{u} / \mu_{N}\right)\right)$ for the $S_{L}^{P}=\frac{1}{2}^{-}\left(\frac{3}{2}^{-}\right)$

\footnotetext{
${ }^{1}$ We notice that there is no ideal choice of a reference molecule, as for no exotic meson there exists a clear consensus of its molecular nature.
} 
$P$-wave charmed mesons $D_{0(1)}^{(*)}\left(D_{1(2)}^{(*)}\right)$, where $\mu_{N}$ is the nuclear magneton, and $\mu_{u} \simeq 1.9 \mu_{N}$ the magnetic moment of a constituent $u$ quark (for a more detailed account on the choice of the magneticlike couplings, we refer to Appendix A). For $g_{S}$ we invoke the linear- $\sigma$ model [30] and the quark model [29], yielding $g_{S} \simeq 3.4$ (6.8) for $D^{(*)}\left(\Sigma_{c}^{(*)}\right)$. For the light-meson masses, we take $m_{S}=$ $475 \mathrm{MeV}$ (the value in the middle of the Review of Particle Physics (RPP) range of 400-550 MeV [31]) and $m_{V}=$ $\left(m_{\rho}+m_{\omega}\right) / 2$ (with $m_{\rho}=770 \mathrm{MeV}$ and $m_{\omega}=780 \mathrm{MeV}$ ).

At this point we find it worth mentioning that the lightmeson exchange picture is not free of theoretical difficulties, the most important of which is probably the nature and width of the $\sigma$. This happens to be a well-known issue for which we briefly review a few of the available solutions in Appendix B. Here it is enough to comment that when a broad meson is exchanged, it can be effectively approximated by a narrow one by a suitable redefinition of its parameters [32,33]. As we are determining the couplings from phenomenological relations, which do not take into account the width of the scalar meson and provide a good description of a few molecular candidates, we consider this redefinition to have already taken place (we refer to Appendix B for further discussion and details).

Regarding uncertainties, the RGE indicates that the value of the saturated couplings are dominated by the scalar meson [see Eq. (11)], which also happens to be the meson for which theoretical uncertainties are larger. For this reason we will generate error bands from the uncertainty in the scalar meson mass, $m_{S}=475 \pm 75 \mathrm{MeV}$. Besides, the contact-range theory approximation also entails uncertainties: if the molecule is compact enough, the heavy hadrons will be able to resolve the details of the interaction binding them, and the contact-range approximation will cease to be valid. We include a relative uncertainty of $\gamma_{\mathrm{mol}} / m_{V}$ (i.e., the ratio of the characteristic molecular momentum scale and the mass of the vector meson) to take into account this effect, which we then sum in quadrature with the previous error coming from $m_{S}$.

With these couplings we now reproduce the $P_{c}(4312)$ with Eq. (14), yielding $C_{P_{c}}^{\text {sat }}=-0.80 \mathrm{fm}^{2}$ for $\Lambda_{H}=1 \mathrm{GeV}$. After this we calculate $R_{\text {mol }}$ and solve Eq. (16) to predict the molecules we show in Table I:

(i) For molecules in the lowest isospin state the general pattern is that mass decreases with light spin [19] (i.e., opposite to compact hadrons).

(ii) The origin of the light-spin dependence is the magneticlike vector-meson exchange term in Eqs. (6) and (9).

(iii) For $\bar{D}^{(*)} \Sigma_{c}^{(*)}$, we reproduce the $P_{c}(4440 / 4457)$ [34] and find the spectrum predicted in Refs. [35-39].

(iv) For $D^{(*)} \bar{D}^{(*)}$, besides the $X$ and its $J^{P C}=2^{++}$ partner $[20,40-42]$, the only other configuration close to binding is the $J^{P C}=0^{++} D \bar{D}$ system $[43,44]$. It should be noticed that:

(a) The isoscalar $D^{(*)} \bar{D}^{(*)}$ systems can mix with nearby charmonia. This is a factor that we have not included in our model, yet discrepancies between our predictions and candidate states could point towards the existence of such mixing.

(b) In particular, the $X(3872)$ is predicted around its experimental mass, though with moderate uncertainties which do not exclude (but do not require either) a charmonium component. Though its nature is still debated [45-49], theoretical works point out to the existence of a nontrivial nonmolecular component (e.g., Ref. [50] finds a negative effective range in the $J^{P C}=1^{++} D^{*} \bar{D}$ system, which is difficult to explain in a purely molecular picture). The previous could be further confirmed if the spectroscopic uncertainties of the present model could be reduced to the point of determining if underbinding or overbinding exist.

(c) Had we used the $X(3872)$ as the reference molecule, the results of Table I would have been almost identical to the ones we obtain from the $P_{c}(4312)$.

(v) Molecules involving $P$-wave $D_{1} / D_{2}^{*}$ mesons have larger hyperfine splittings than their $D / D^{*}$ counterparts.

(a) For instance, the $D_{1(2)}^{(*)} \bar{D}_{1(2)}^{(*)}$ molecules only bind for configurations with high light spin, while the configurations with low light-spin content can even become repulsive.

(b) The hyperfine splitting of the $\Sigma_{c} \bar{D}_{1}$ pentaquarks is predicted to be approximately twice as large as for the $\Sigma_{c} \bar{D}^{*}$ one, about $30 \mathrm{MeV}$ instead of $15 \mathrm{MeV}$.

(vi) Molecules for which rho and omega exchange cancel out $\left(Z_{c}(3900)\right.$ [51]) or involving strangeness $\left(P_{c s}(4459)[52]\right)$ require additional discussion and are not listed. We advance that:

(a) For the $Z_{c}$ [53-56] $I^{G}\left(J^{P C}\right)=1^{+}\left(1^{+-}\right) D^{*} \bar{D}$ configuration, we predict a virtual state at $M=3849.6 \mathrm{MeV}$. This agrees with the effective field theory (EFT) analysis of Ref. [57], which suggests that if the $Z_{c}$ is a virtual state, then its mass would be in the $M=(3831-3844) \mathrm{MeV}$ range, though with large uncertainties. Meanwhile in the EFT approach of Ref. [53] the $Z_{c}$ is located at (3867-3871) MeV when a virtual state solution is assumed (fit 1 in Ref. [53]). Recently, Ref. [58] has proposed the inclusion of axial meson $\left[a_{1}(1260)\right]$ exchange to explain the $Z_{c}$, in which case we would end up with a mass in the (3856-3867) $\mathrm{MeV}$ range. However, this depends on the assumption that Eq. (11) holds far 
away from $\Lambda=m_{V}$, which might very well not be correct without suitable modifications (check the discussion in Appendix B). For comparison, Ref. [18] explains the $Z_{c}$ states in terms of vector charmonia exchanges.

(b) If the scalar meson couples to $q=u, d, s$ with similar strength [58], then this will generate $P_{c s^{-}}$ like [59-62] $I=0, J=\frac{1}{2}, \frac{3}{2} \Xi_{c} \bar{D}^{*}$ bound states at $M=4466.9 \mathrm{MeV}$.

(vii) We remind that the results of Table I ignore pion exchanges and coupled channel effects, which are considered to be perturbative corrections. Appendices $\mathrm{C}$ and $\mathrm{D}$ explicitly check these two assumptions in a few concrete cases, leading, in general, to corrections that are indeed smaller than the uncertainties shown in Table I. We notice that there might be specific molecules for which these assumptions do not hold.

Finally, we warn that though the formalism is identical to the one used in a typical contact-range EFT, this is not EFT: here the cutoff is not "auxiliary" but physical. It is not expected to run freely but a parameter chosen to reproduce the known spectrum.

\section{V. $S$-TO-P-WAVE CHARMED MESON TRANSITIONS}

Now we want to explore the $Y(4260)$ [65], which has been conjectured to be a $J^{P C}=1^{--} D \bar{D}_{1}$ molecule [6671], though its nature remains unclear [72-77].

For the $D_{1(2)}^{(*)} \bar{D}^{(*)}$ two-hadron system, the electric dipolar and magnetic quadrupolar $D_{1(2)}^{(*)} \rightarrow D^{(*)}$ transitions are possible, i.e., there are new $\mathrm{H}_{1} \mathrm{H}_{2} \rightarrow \mathrm{H}_{2} \mathrm{H}_{1}$ components in the potential not present in Eq. (1), which we write as

$V_{C}=C_{0}+C_{1} \vec{\sigma}_{L 1} \cdot \hat{\vec{S}}_{L 2}+C_{1}^{\prime} \vec{\Sigma}_{L 1}^{\dagger} \cdot \vec{\Sigma}_{L 2}+C_{2}^{\prime} Q_{L 1 i j}^{\dagger} Q_{L 2 i j}$,

where $\vec{\sigma}_{L 1}$ are the Pauli matrices and $\hat{\vec{S}}_{L 2}$ the spin $-\frac{3}{2}$ matrices, as applied to the light quark within the $D_{1(2)}^{(*)}$. The dipolar and quadrupolar pieces are described by the $C_{1}^{\prime}$ and $C_{2}^{\prime}$ couplings, $\vec{\Sigma}_{L} / \vec{\Sigma}_{L}^{\dagger}$ are the spin matrices for the $S_{L}=\frac{1}{2}$ to $\frac{3}{2}$ transition (which can be consulted in the appendices of Ref. [21]), and $Q_{L i j}=\left(\sigma_{L i} \Sigma_{L j}+\sigma_{L j} \Sigma_{L i}\right) / 2$. For saturating $C_{1}^{\prime}$ and $C_{2}^{\prime}$, we consider the Lagrangians,

$$
\begin{array}{r}
\mathcal{L}_{E 1}=\frac{f_{V}^{\prime}}{2 M} q_{L}^{\dagger} \Sigma_{L i}\left(\partial_{i} V_{0}-\partial_{0} V_{i}\right) q_{L}^{\prime}+\text { C.C. }, \\
\mathcal{L}_{M 2}=\frac{h_{V}^{\prime}}{(2 M)^{2}} q_{L}^{\dagger} Q_{L i j} \partial_{i}\left(\epsilon_{j l m} \partial_{l} V_{m}\right) q_{L}^{\prime}+\text { C.C. }
\end{array}
$$

where $q_{L}$ and $q_{L}^{\prime}$ are the nonrelativistic light-quark subfields for the $S$ - and $P$-wave charmed mesons, generating the potentials

$$
\begin{gathered}
V_{E 1}=-\frac{f_{V}^{\prime 2}}{4 M^{2}} \frac{\omega_{V}^{2}+\frac{1}{3} \mu_{V}^{2}}{\vec{q}^{2}+\mu_{V}^{2}} \vec{\Sigma}_{L 1}^{\dagger} \cdot \vec{\Sigma}_{L 2}+\ldots \\
V_{M 2}=-\frac{h_{V}^{\prime 2}}{16 M^{4}} \frac{1}{5} \frac{\mu_{V}^{4}}{\vec{q}^{2}+\mu_{V}^{2}} Q_{L 1 i j}^{\dagger} Q_{L 2 i j}+\ldots
\end{gathered}
$$

where $\mu_{V}^{2}=m_{V}^{2}-\omega_{V}^{2}$ is the effective vector-meson mass for this transition, and $\omega_{V}=m\left(D_{1}\right)-m(D)$, $m\left(D_{2}^{*}\right)-m(D), m\left(D_{1}\right)-m\left(D^{*}\right)$ or $m\left(D_{2}^{*}\right)-m\left(D^{*}\right)$ for $D_{1} \bar{D}, D_{2}^{*} \bar{D}, D_{1} \bar{D}^{*}$ or $D_{2}^{*} \bar{D}^{*}$ molecules. The saturated couplings read

$$
\begin{array}{r}
C_{1}^{\text {sat }}\left(m_{V}\right) \propto-\frac{f_{V}^{\prime 2}}{4 M^{2}}\left(\frac{m_{V}}{\mu_{V}}\right)^{\alpha}\left(\frac{\omega_{V}^{2}+\frac{1}{3} \mu_{V}^{2}}{\mu_{V}^{2}}\right)\left[\zeta+\hat{\vec{I}}_{1} \cdot \hat{\vec{I}}_{2}\right], \\
C_{2}^{\text {sat }}\left(m_{V}\right) \propto-\frac{h_{V}^{\prime 2}}{16 M^{4}}\left(\frac{m_{V}}{\mu_{V}}\right)^{\alpha} \frac{\mu_{V}^{2}}{5}\left[\zeta+\hat{\vec{I}}_{1} \cdot \hat{\vec{I}}_{2}\right],
\end{array}
$$

which includes the RGE correction derived in Eqs. (10)-(12). If we define $f_{V}^{\prime}=\kappa_{E 1}^{\prime} g_{V}$, we note that $\kappa_{E 1}^{\prime}$ can be determined from Eq. (17) and the $D_{1(2)}^{(*)} \rightarrow$ $D^{(*)} \gamma$ dipolar moment (extractable from the partial decay widths [78] or the $\left\langle D_{1(2)}^{(*)}|r| D^{(*)}\right\rangle$ matrix elements [79]), yielding $\kappa_{E 1}^{\prime} \sim(2.6-3.6)$. This provides a fairly strong attraction in the $J^{P C}=1^{--} D \bar{D}_{1}$ molecular configuration, for which $\vec{\Sigma}_{L 1}^{\dagger} \cdot \vec{\Sigma}_{L 2}=-1$ (while $Q_{L 1}^{\dagger} \cdot Q_{L 2} \equiv Q_{L 1 i j}^{\dagger} Q_{L 2 i j}=0$ ), resulting in $B_{\mathrm{mol}}=19-55 \mathrm{MeV}$ and a mass of $4235-4271 \mathrm{MeV}$, to be compared with $4218.6 \pm 5.2$ [64] for the $Y(4260)\left(\kappa_{E 1}^{\prime} \sim 3.9\right.$ would reproduce the mass), suggesting a sizable molecular component.

For the magnetic quadrupolar term, we define $h_{V}^{\prime}=\kappa_{M 2}^{\prime} g_{V}$, where $\kappa_{M 2}^{\prime}$ can be extracted from $\kappa_{E 1}^{\prime}$ by the relation $\kappa_{M 2}^{\prime}=\left(m_{N} / m_{q}\right) \kappa_{E 1}^{\prime}=7.4-15.4$, with $m_{N} / m_{q}$ the ratio of the nucleon and constituent quark masses in the particular quark-model calculation used to obtain $\kappa_{M 2}^{\prime}$ (see Appendix A for details). This provides a modest (but sizable) attraction in the $J^{P C}=1^{--} D^{*} \bar{D}_{1}$ system (where $\vec{\Sigma}_{L 1}^{\dagger} \cdot \vec{\Sigma}_{L 2}=-\frac{1}{6}$ and $Q_{L 1}^{\dagger} \cdot Q_{L 2}=-\frac{5}{4}$ ), resulting in a virtual state with $B_{\text {mol }}^{V}=4.1 \mathrm{MeV}$ for $\kappa_{E 1}^{\prime}=3.1$ and $\kappa_{M 2}^{\prime}=10.7$. If we consider the uncertainties in the M1 and E2 couplings, then the end result ranges from a shallow bound state $\left(B_{\text {mol }}=1.1 \mathrm{MeV}\right)$ to a virtual state moderately away from threshold $\left(B_{\mathrm{mol}}^{V}=16.4 \mathrm{MeV}\right)$, while for $\kappa_{E 1}^{\prime}=3.9$ [which reproduces the $Y(4260)$ ] and $\kappa_{M 2}^{\prime}=24.6$, the location of the $Y(4360)$ as recently measured by BESIII [64] would be reproduced. Yet, the previous explanation does not consider the possible $D^{*} \bar{D}_{1}-D^{*} \bar{D}_{1}^{*}$ coupled channel effects (the $D_{1}$ and $D_{1}^{*}$ charmed mesons have 
about the same mass), which would make binding more likely and explain the larger width of the $Y(4360)$, check Appendix D 4 for details. Thus, while a pure molecular explanation of the $Y(4360)$ is less natural than for the $Y(4260)$, a molecular component is nonetheless possible and maybe even expectable.

Two other interesting configurations are the $J^{P C}=$ $2^{--} D \bar{D}_{2}^{*}$ and $3^{--} D^{*} \bar{D}_{2}^{*}$ systems: the first depends on the coupling $C_{2}^{\prime}$, but not on $C_{1}^{\prime}\left(\vec{\Sigma}_{L 1}^{\dagger} \cdot \vec{\Sigma}_{L 2}=0\right.$ and $Q_{L 1}^{\dagger} \cdot Q_{L 2}=-1$ ), which means that if observed it could be used to determine $\kappa_{M 2}^{\prime}$. For $\kappa_{M 2}^{\prime}=7.4-15.4$, we predict a binding energy and mass of $B_{\mathrm{mol}}=(0.1-9.1) \mathrm{MeV}$ and $M=(4319.3-4327.5) \mathrm{MeV}$. The second happens to be the most attractive configuration $\left(\vec{\Sigma}_{L 1}^{\dagger} \cdot \vec{\Sigma}_{L 2}=-1\right.$ and $Q_{L 1}^{\dagger} \cdot Q_{L 2}=-\frac{1}{2}$ ), with a state predicted somewhere in the $M=(4349-4401) \mathrm{MeV}$ window. In Table I we list the central value predictions (i.e., $\kappa_{E 1}^{\prime}=3.1$ and $\kappa_{M 2}^{\prime}=10.7$ ) for the four $S$ - and $P$-wave molecules considered here.

\section{LIGHT BARYONS}

For baryons containing only light quarks, the previous ideas can also be applied. However, there is a tweak which has to do with the relative sizes of light baryons in comparison with heavy hadrons: light hadrons are larger than heavy hadrons. For instance, the electromagnetic radius $\sqrt{\left\langle r^{2}\right\rangle_{\text {e.m. }}}$ of the charged pion and kaon are about 0.66 and $0.56 \mathrm{fm}$, respectively [31], with size decreasing once the heavier strange quark is involved. This pattern also applies to the charmed mesons, for which $\sqrt{\left\langle r^{2}\right\rangle_{\text {e.m. }}} \sim(0.40-0.55)$ fm [80-83]. For baryons, the electromagnetic radius of the proton is $0.84 \mathrm{fm}$ [31]. Lattice QCD calculations of the electromagnetic form factors of the singly and doubly charmed baryons yield figures of the order of 0.5 and $0.4 \mathrm{fm}$, respectively [84,85], half the proton radius, where it is curious to notice that the doubly charmed baryons are about the same size as the charmed mesons and only slightly smaller than the singly charmed ones (from which the hypothesis of using the same cutoff for all heavy hadrons seems a sensible choice).

Of course, the problem is how to take this effect into account. The easiest idea is to use a softer physical cutoff (which effectively amounts to the introduction of a new parameter) in the light baryon sector,

$$
\left\langle p^{\prime}\left|V_{C}\right| p\right\rangle=C_{\mathrm{mol}}^{\mathrm{sat}} f\left(\frac{p^{\prime}}{\Lambda_{\mathrm{L}}}\right) f\left(\frac{p}{\Lambda_{\mathrm{L}}}\right)
$$

where $\Lambda_{\mathrm{L}}$ will be close to $\Lambda_{H} / 2$, with $C_{\mathrm{mol}}^{\text {sat }}$ still determined as a ratio of $C_{P_{c}}^{\text {sat }}$. For nucleons $g_{S}=10.2, \frac{1}{3} g_{\omega}=g_{\rho}=g$ and $\kappa_{\rho}=3.7, \kappa_{\omega}=-0.1$ [86], from which we get

$$
R_{\text {singlet }}=1.77, \quad R_{\text {triplet }}=1.86,
$$

which are really similar, reproducing Wigner's SU(4) symmetry [87-89]. For $\Lambda_{L}=0.5 \mathrm{GeV}$, we predict shallow singlet/triplet bound states

$$
B_{\text {singlet }}=0.20 \mathrm{MeV}, \quad B_{\text {triplet }}=0.94 \mathrm{MeV},
$$

i.e., close to reality, where the singlet/triplet is a virtual/ bound state located at $0.07 / 2.22 \mathrm{MeV}$ below threshold. It is intriguing how reproducing the deuteron forces us to choose a $\Lambda_{L}$ that basically coincides with the scale at which Wigner's symmetry is expected to manifest [90-94]. Even though our choice of $\Lambda_{L}$ follows from a phenomenological argument, in practice, this is a new parameter required for the correct description of systems containing a light baryon, and it could have as well been determined from the condition of reproducing the deuteron or the virtual state.

Another system to consider is $\Delta \Delta$, with $g_{\rho}=g_{\omega}=3 g$ and $\kappa_{\rho}=\kappa_{\omega}=\frac{3}{2} \mu_{u}$. The most attractive configuration is $I=0$ and $S=3\left(B_{\Delta \Delta}=15 \mathrm{MeV}\right.$ and $M_{\Delta \Delta}=2405 \mathrm{MeV}$ for $M_{\Delta} \simeq 1210 \mathrm{MeV}$ [31]), which might be identified with the hexaquark predicted six decades ago [95], the $d^{*}(2380)$ observed in [96] (which, however, has recently been argued to be a triangle singularity [97]) or the $\Delta \Delta$ state computed in the lattice [98].

\section{LIGHT-HEAVY SYSTEMS}

Finally, for a two-hadron system with a light and heavy hadron, we simply use different cutoffs for each hadron,

$$
\left\langle p^{\prime}\left|V_{C}\right| p\right\rangle=C_{\text {mol }}^{\text {sat }} f\left(\frac{p^{\prime}}{\Lambda_{\mathrm{L}}}\right) f\left(\frac{p}{\Lambda_{\mathrm{H}}}\right)
$$

If we apply this idea to the $N D^{*}$ system, we find a bound state with $I\left(J^{P}\right)=0\left(\frac{3}{2}^{-}\right)$that might correspond to the $\Lambda_{c}(2940)$, which has been theorized to be molecular [99-104]. We also find it worth mentioning the prediction of a virtual state in the $N D$ system with $I\left(J^{P}\right)=1\left(\frac{1}{2}^{-}\right)$, $B_{\text {mol }}^{V}=4.3 \mathrm{MeV}$ and $M=2806.6 \mathrm{MeV}$, which might be identified with the $\Sigma_{c}(2800)$ (also theorized to be molecular $[104,105])$. However, owing to the $\Sigma_{c}(2800)$ having being observed in the $\Lambda_{c} \pi$ spectrum [106], this interpretation is questionable unless it is only partially molecular or there are other factors increasing the attraction in the $I=1 \mathrm{ND}$ system. We notice though that Ref. [104] finds a $\Sigma_{c}(2800)$ resonance as a pole in $N D$ scattering. In contrast, the $N \bar{D}^{(*)}$ system (i.e., singly charmed pentaquark candidates) shows less attraction than the $N D^{(*)}$, owing to omega exchange becoming repulsive. Yet, the $I\left(J^{P}\right)=0\left(\frac{3}{2}^{-}\right) N \bar{D}^{*}$ configuration happens to be close to binding, with a virtual state at $B_{\mathrm{mol}}^{V}=1.8 \mathrm{MeV}$. 
TABLE I. Selection of the molecular states predicted from saturation: "System" is the two-hadron system, $I\left(J^{P(C)}\right)$ refers to the isospin, angular momentum, parity, and $C$-parity (if applicable) of the state, $R_{\text {mol }}$ is the relative interaction strength with respect to the $P_{c}$ (4312) [see Eq. (15)], $B_{\text {mol }}$ the binding energy in $\mathrm{MeV}$ (where $(\ldots)^{V}$ indicates a virtual state), $M_{\text {mol }}$ the mass of the molecule, "Candidate" refers to known resonances that might be identified with the predicted molecule, and $M_{\text {candidate }}$ is the candidate's mass (" $1 S_{0}$ pole" refers to the virtual state in singlet nucleon-nucleon scattering). The binding energies are calculated from Eqs. (14)-(16), where for light- (heavy-) hadrons vertices we use the cutoff $\Lambda_{L}=0.5 \mathrm{GeV}\left(\Lambda_{H}=1.0 \mathrm{GeV}\right)$. The uncertainty in $R_{\text {mol }}$ is obtained from varying the $m_{\sigma}$ within the 400-550 MeV range, while for $B_{\text {mol }}$ we combine the previous uncertainty with a $\gamma_{\text {mol }} / m_{V}$ relative error by summing them in quadrature. For the hadron masses, we use the isospin averages of the RPP values [31]. The masses for the $\Lambda_{c}(2765), \Lambda_{c}(2940)$, $\Sigma_{c}(2800)$, and $X(3872)$ are taken from the RPP [31] (we notice though that the $\Lambda_{c}(2765)$ is not well established and could even be a $\Sigma_{c^{-}}$ type state or a superposition of two states instead, though Ref. [63] considers it to be a $\Lambda_{c}$ ); for the $Y(4260)$ and $Y(4360)$, we use the recent BESIII measurements [64], and for the $P_{c}(4312 / 4440 / 4457)$, we refer to the original LHCb observation [34].

\begin{tabular}{|c|c|c|c|c|c|c|}
\hline System & $I\left(J^{P(C)}\right)$ & $R_{\text {mol }}$ & $B_{\text {mol }}$ & $M_{\mathrm{mol}}$ & Candidate & $M_{\text {candidate }}$ \\
\hline$n p$ & $0\left(1^{+}\right)$ & $1.66_{-0.14}^{+0.12}$ & $0.9_{-0.8}^{+1.2}$ & $1876.9_{-1.2}^{+0.8}$ & Deuteron & 1875.6 \\
\hline$n p$ & $1\left(0^{+}\right)$ & $1.54_{-0.20}^{+0.16}$ & $0.2_{-0.4}^{+1.1}$ & $1877.7_{-1.1}^{+0.2}$ & ${ }^{1} S_{0}$ pole & 1877.8 \\
\hline$N D$ & $0\left(\frac{1}{2}^{-}\right)$ & $1.01_{-0.05}^{+0.06}$ & $\left(0.7_{-0.5}^{+0.6}\right)^{V}$ & $2805.5_{-0.6}^{+0.5}$ & $\Lambda_{c}(2765)$ & 2766.6 \\
\hline$N D^{*}$ & $0\left(\frac{3}{2}^{-}\right)$ & $1.19_{-0.11}^{+0.12}$ & $0.1_{-0.2}^{+0.9}$ & $2947.4_{-0.9}^{+0.1}$ & $\Lambda_{c}(2940)$ & 2939.6 \\
\hline$N D$ & $1\left(\frac{1}{2}^{-}\right)$ & $0.84_{-0.02}^{+0.02}$ & $\left(4.3_{-0.6}^{+0.7}\right)^{V}$ & $2801.9_{-0.7}^{+0.6}$ & $\Sigma_{c}(2800)$ & $\sim 2800$ \\
\hline$N D^{*}$ & $1\left(\frac{1}{2}^{-}\right)$ & $0.97_{-0.02}^{+0.03}$ & $\left(1.2_{-0.4}^{+0.4}\right)^{V}$ & $2946.3_{-0.4}^{+0.4}$ & $\ldots$ & $\ldots$ \\
\hline$N \bar{D}^{*}$ & $0\left(\frac{3}{2}^{-}\right)$ & $0.94_{-0.01}^{+0.01}$ & $\left(1.8_{-0.2}^{+0.3}\right)^{V}$ & $2945.7_{-0.3}^{+0.2}$ & $\ldots$ & $\ldots$ \\
\hline$D \bar{D}$ & $0\left(0^{++}\right)$ & $0.63_{-0.07}^{+0.08}$ & $\left(1.5_{-1.5}^{+3.5}\right)^{V}$ & $3733.0_{-3.5}^{+1.5}$ & $\ldots$ & $\ldots$ \\
\hline$D^{*} \bar{D}$ & $0\left(1^{++}\right)$ & $0.89_{-0.16}^{+0.20}$ & $4.1_{-4.1}^{+11.6}$ & $3871.7_{-11.6}^{+4.1}$ & $X(3872)$ & 3871.69 \\
\hline$D^{*} \bar{D}^{*}$ & $0\left(2^{++}\right)$ & $0.93_{-0.17}^{+0.20}$ & $5.5_{-5.8}^{+12.6}$ & $4011.6_{-12.6}^{+5.5}$ & $\cdots$ & $\ldots$ \\
\hline$D_{1} \bar{D}$ & $0\left(1^{--}\right)$ & $1.33_{-0.31}^{+0.36}$ & $34_{-26}^{+30}$ & $4255_{-30}^{+26}$ & $Y(4260)$ & 4218.6 \\
\hline$D_{2} \bar{D}$ & $0\left(2^{--}\right)$ & $0.87_{-0.13}^{+0.15}$ & $2.7_{-2.8}^{+7.3}$ & $4325.6_{-7.3}^{+2.8}$ & $\ldots$ & $\ldots$ \\
\hline$D_{1} \bar{D}^{*}$ & $0\left(1^{--}\right)$ & $0.56_{-0.02}^{+0.02}$ & $\left(4.1_{-1.1}^{+1.1}\right)^{V}$ & $4425.6_{-1.1}^{+1.1}$ & $Y(4360)$ & 4382.0 \\
\hline$D_{2} \bar{D}^{*}$ & $0\left(3^{--}\right)$ & $1.89_{-0.51}^{+0.60}$ & $90_{-73}^{+90}$ & $4380_{-90}^{+73}$ & $\ldots$ & $\ldots$ \\
\hline$D_{1} \bar{D}_{1}$ & $0\left(2^{++}\right)$ & $1.66_{-0.40}^{+0.47}$ & $58_{-45}^{+57}$ & $4786_{-57}^{+45}$ & $\ldots$ & $\ldots$ \\
\hline$D_{2} \bar{D}_{1}$ & $0\left(3^{+-}\right)$ & $1.64_{-0.39}^{+0.46}$ & $56_{-43}^{+55}$ & $4829_{-55}^{+43}$ & $\ldots$ & $\ldots$ \\
\hline$D_{2} \bar{D}_{1}$ & $0\left(3^{++}\right)$ & $2.04_{-0.58}^{+0.65}$ & $97_{-81}^{+95}$ & $4788_{-95}^{+81}$ & $\ldots$ & $\ldots$ \\
\hline$D_{2} \bar{D}_{2}$ & $0\left(3^{+-}\right)$ & $0.83_{-0.09}^{+0.11}$ & $1.4_{-1.5}^{+3.6}$ & $4924.7_{-3.6}^{+1.4}$ & $\ldots$ & $\ldots$ \\
\hline$D_{2} \bar{D}_{2}$ & $0\left(4^{++}\right)$ & $2.06_{-0.54}^{+0.65}$ & $98_{-82}^{+96}$ & $4828_{-96}^{+82}$ & $\ldots$ & $\ldots$ \\
\hline$\Sigma_{c} \bar{D}$ & $\frac{1}{2}\left(\frac{1}{2}^{-}\right)$ & 1.00 & 8.9 & 4311.9 & $P_{c}(4312)$ & 4311.9 \\
\hline$\Sigma_{c}^{*} \bar{D}$ & $\frac{1}{2}\left(\frac{3}{2}^{-}\right)$ & 1.04 & $9.4_{-1.7}^{+1.7}$ & $4376.0_{-1.7}^{+1.7}$ & $\ldots$ & $\ldots$ \\
\hline$\Sigma_{c} \bar{D}^{*}$ & $\frac{1}{2}\left(\frac{1}{2}^{-}\right)$ & $0.85_{-0.12}^{+0.06}$ & $2.3_{-1.9}^{+2.6}$ & $4459.8_{-2.9}^{+2.3}$ & $P_{c}(4457)$ & 4457.3 \\
\hline$\Sigma_{c} \bar{D}^{*}$ & $\frac{1}{2}\left(\frac{3}{2}^{-}\right)$ & $1.13_{-0.03}^{+0.04}$ & $16.9_{-4.7}^{+5.1}$ & $4445.2_{-5.1}^{+4.7}$ & $P_{c}(4440)$ & 4440.3 \\
\hline$\Sigma_{c}^{*} \bar{D}^{*}$ & $\frac{1}{2}\left(\frac{1}{2}^{-}\right)$ & $0.82_{-0.04}^{+0.09}$ & $1.3_{-1.3}^{+2.7}$ & $4525.4_{-2.7}^{+1.3}$ & $\ldots$ & $\ldots$ \\
\hline$\Sigma_{c}^{*} \bar{D}^{*}$ & $\frac{1}{2}\left(\frac{3}{2}^{-}\right)$ & $0.96_{-0.04}^{+0.03}$ & $6.4_{-2.0}^{+2.0}$ & $4520.3_{-2.0}^{+2.0}$ & $\ldots$ & $\ldots$ \\
\hline$\Sigma_{c}^{*} \bar{D}^{*}$ & $\frac{1}{2}\left(\frac{5}{2}^{-}\right)$ & $1.19_{-0.05}^{+0.06}$ & $21.0_{-6.8}^{+7.5}$ & $4505.7_{-7.5}^{+6.8}$ & $\ldots$ & $\ldots$ \\
\hline$\Sigma_{c} \bar{D}_{1}$ & $\frac{1}{2}\left(\frac{1}{2}^{+}\right)$ & $0.81_{-0.15}^{+0.14}$ & $1.0_{-1.4}^{+3.8}$ & $4874.6_{-3.8}^{+1.0}$ & $\ldots$ & $\ldots$ \\
\hline$\Sigma_{c} \bar{D}_{1}$ & $\frac{1}{2}\left(\frac{3}{2}^{+}\right)$ & $1.32_{-0.06}^{+0.07}$ & $29_{-11}^{+12}$ & $4847_{-12}^{+11}$ & $\ldots$ & $\ldots$ \\
\hline$\Sigma_{c} \bar{D}_{2}^{*}$ & $\frac{1}{2}\left(\frac{3}{2}^{+}\right)$ & $0.75_{-0.18}^{+0.14}$ & $0.1_{-3.4}^{+3.3}$ & $4916.5_{-3.3}^{+0.1}$ & $\ldots$ & $\ldots$ \\
\hline$\Sigma_{c} \bar{D}_{2}^{*}$ & $\frac{1}{2}\left(\frac{5}{2}^{+}\right)$ & $1.43_{-0.10}^{+0.12}$ & $37_{-17}^{+18}$ & $4879_{-18}^{+17}$ & $\cdots$ & $\cdots$ \\
\hline
\end{tabular}

\section{CONCLUSIONS}

We propose a description of heavy- (and light-)hadron molecules in terms of an $S$-wave contact-range potential. The couplings of this potential are determined from light-meson exchange by means of a saturation procedure, which incorporates a few RG ideas to effectively combine together the contribution from scalar and vector mesons. In turn the light-meson exchange parameters are set from a 
series of well-known phenomenological ideas, and a cutoff $\Lambda_{H} \sim 1 \mathrm{GeV}$ is included. This procedure takes the $P_{c}(4312)$ as input, from which it is able to reproduce the other two LHCb pentaquarks, the $X(3872)$ and predict a few new molecular candidates. If applied to the light sector (with a few modifications), it reproduces Wigner-SU(4) symmetry and the deuteron as a shallow bound state. Of course, the question is whether the theoretical ideas contained in this manuscript do really represent a good approximation to the spectrum of molecular states. Future experiments will tell, particularly the discovery of different spin configurations of a given two-hadron system, as the hyperfine splittings are very dependent on their origin, which we conjecture to be the magneticlike couplings of the vector mesons.

\section{ACKNOWLEDGMENTS}

We thank Feng-Kun Guo, Enrique Ruiz Arriola, and Eulogio Oset for their comments on this manuscript. M.P. V. thanks the IJCLab of Orsay, where part of this work was done, for its hospitality. This work is partly supported by the National Natural Science Foundation of China under Grants No. 11735003 and 11975041, the Fundamental Research Funds for the Central Universities, and the Thousand Talents Plan for Young Professionals.

Note added.-After the acceptance of this manuscript, the ALICE collaboration has presented the first experimental study of the $N \bar{D}$ interaction [107]. They extract the isoscalar $(I=0) N \bar{D}$ inverse scattering length $\left(f_{0}^{-1}\right.$ in their notation), resulting in $f_{0}^{-1}(I=0) \in[-0.4,0.9] \mathrm{fm}^{-1}$. Our own calculation yields $f_{0}^{-1}(I=0)=0.64_{-0.15}^{+0.19} \mathrm{fm}^{-1}$ (indicating the presence of a virtual state), or $[0.49,0.83] \mathrm{fm}^{-1}$, a range which falls within the ALICE estimation.

\section{APPENDIX A: DETERMINATION OF THE M1, E1, AND M2 COUPLINGS}

Here we briefly explain how we derive the vector-meson couplings of higher polarity, beginning with the magnetic M1 $\kappa_{V}$ couplings. These are given by $\kappa_{V}=\frac{3}{2} \mu_{u}(j, l) / \mu_{N}$, with $\mu_{u}(j, l)$ the magnetic moment of a light $u$ quark with total and orbital angular momentum $j$ and $l$ (which also characterize its parent heavy meson). Within the quark model we expect the light-quark magnetic moment operator to be

$$
\hat{\mu}_{q}=\frac{e_{q}}{2 m_{q}}\left[\vec{\sigma}_{q}+\vec{l}_{q}\right]=\mu_{q}\left[\vec{\sigma}_{q}+\vec{l}_{q}\right]
$$

where $\mu_{q}$ is the magnetic moment of the $q=u, d$ quarks in the quark model $\left(\mu_{u} \simeq 1.9 \mu_{N}\right.$ and $\left.\mu_{d} \simeq-0.9 \mu_{N}\right), \vec{\sigma}_{q}$ the Pauli matrices as applied to the intrinsic spin of the light quark, and $\vec{l}_{q}$ the light-quark orbital angular momentum operator. For a light quark with $j$ and $l$ quantum numbers, its magnetic moment is given by the matrix element

$$
\mu_{q}(j, l)=\left\langle(s l) j j\left|\hat{\mu}_{q}\right|(s l) j j\right\rangle,
$$

with $|(s l) j j\rangle$ a state in which a light quark with spin $s=\frac{1}{2}$ and orbital angular momentum $l$ couples to total angular momentum $j$ and third component $j$. The calculation of this matrix element is trivial, yielding

$$
\begin{gathered}
\mu_{q}\left(j=l+\frac{1}{2}, l\right)=\mu_{q}\left(j+\frac{1}{2}\right), \\
\mu_{q}\left(j=l-\frac{1}{2}, l\right)=\mu_{q} \frac{j}{j+1}\left(j+\frac{1}{2}\right),
\end{gathered}
$$

which translates into $\mu_{u}\left(\frac{1}{2}, 1\right)=\mu_{u} / 3$ and $\mu_{u}\left(\frac{3}{2}, 1\right)=2 \mu_{u}$ for the $D_{0} / D_{1}^{*}$ and $D_{1} / D_{2}^{*} P$-wave charmed mesons.

The E1 and M2 couplings $\kappa_{E 1}^{\prime}$ and $\kappa_{M 2}^{\prime}$, which determine the strength of the $P$-to- $S$-wave charmed meson transitions, can be determined in turn from the electric dipolar and magnetic quadrupolar moments of these transitions. A comparison with the E1 and M2 electromagnetic Lagrangians,

$$
\begin{gathered}
\mathcal{L}_{E 1}=\left\langle e_{q}\right\rangle d_{E}^{\prime} q_{L}^{\dagger} \vec{\Sigma}_{L} \cdot\left(\partial_{0} \vec{A}-\vec{\partial} A_{0}\right) q_{L}^{\prime}+\text { C.C. } \\
\mathcal{L}_{M 2}=Q_{M}^{q} q_{L}^{\dagger} Q_{L i j} \partial_{i} B_{j} q_{L}^{\prime}+\text { C.C. }
\end{gathered}
$$

together with Eq. (17) yields $\kappa_{E 1}^{\prime}=2 M \frac{3}{2}\left\langle e_{u}\right\rangle d_{E}^{\prime}$ and $\kappa_{M 2}^{\prime}=(2 M)^{2} \frac{3}{2} Q_{M}^{u}$, where in the Lagrangians above $q_{L}$, $q_{L}^{\prime}, \vec{\Sigma}_{L}$ and $Q_{L i j}$ are defined as below Eqs. (19) and (20), $\left\langle e_{q}\right\rangle=\left(m_{Q} e_{q}-m_{q} e_{\bar{Q}}\right) /\left(m_{q}+m_{Q}\right)$ is the effective charge of the $q \bar{Q}$ system $\left(\left\langle e_{u}\right\rangle=\frac{2}{3}\right.$ for charmed antimesons), $Q_{M}^{q}$ is the quadrupolar magnetic moment for the light quark, $q=u, \quad d, \quad A_{\mu}=\left(A_{0}, \vec{A}\right)$ the photon field, and $B_{j}=$ $\epsilon_{j l m} \partial_{l} A_{m}$ the magnetic field.

If we begin with the E1 transitions, $\left\langle e_{q}\right\rangle d_{E}^{\prime}$ is the electric dipolar moment of the $u$ quark in the $D_{1(2)}^{(*)} \rightarrow D^{(*)}$ transition. The dipolar moment can in turn be obtained in two different ways: (i) from the matrix elements of the dipolar moment operator or (ii) from the $D_{1(2)}^{*} \rightarrow D^{(*)} \gamma$ decays. In the first case we consider the operator

$$
\hat{d}_{E}^{q}=\left\langle e_{q}\right\rangle \vec{r}
$$

where we define the dipolar moment in relation with the matrix element

$$
\left\langle D_{1(2)}^{(*)}\left|\hat{d}_{E}^{q}\right| D^{(*)}\right\rangle=\left\langle e_{q}\right\rangle d_{E}^{\prime} \vec{\Sigma}_{L} .
$$

We calculate $d_{E}^{\prime}$ from the quark-model wave functions of the $S_{L}=\frac{1}{2}$ or $\frac{3}{2}$ charmed meson, which can be expanded as

$$
\left|D\left(S_{L} ; J M\right)\right\rangle=\sum_{M_{L} M_{H}} \Psi_{S_{L} M_{L}}(\vec{r})\left|S_{H} M_{H}\right\rangle\left\langle S_{L} M_{L} S_{H} M_{H} \mid J M\right\rangle,
$$


$\Psi_{S_{L} M_{L}}(\vec{r})=\sum_{\mu_{l} \mu_{s}} \frac{u_{l}(r)}{r} Y_{l \mu_{l}}(\hat{r})\left|s \mu_{s}\right\rangle\left\langle l \mu_{l} s \mu_{s} \mid S_{L} M_{L}\right\rangle$,

where $J, M$ refer to the total angular momentum of the charmed meson and its third component, $S_{L}, M_{L}$ and $S_{H}\left(=\frac{1}{2}\right), M_{H}$ to the light- and heavy-quark spin, $\Psi_{S_{L} M_{L}}$ the wave function of the light quark, $l, \mu_{l}$ and $s\left(=\frac{1}{2}\right), \mu_{s}$ the orbital and intrinsic angular momentum of the light quark, $u_{l}$ the reduced wave function, $Y_{l \mu_{l}}$ a spherical harmonic, and $|j m\rangle$ and $\left\langle j_{1} m_{1} j_{2} m_{2} \mid j m\right\rangle$ refer to spin wave functions and Clebsch-Gordan coefficients. After a few manipulations, we arrive at

$$
d_{E}^{\prime}=-\frac{\langle P|r| S\rangle}{\sqrt{3}},
$$

where

$$
\langle P|r| S\rangle=\int_{0}^{\infty} d r u_{P}(r) r u_{S}(r),
$$

with $u_{S}$ and $u_{P}$ the $l=0,1$ reduced wave functions. We find that $\kappa_{E 1}^{\prime}=-2 m_{N} d_{E}^{\prime}$ (for $M=m_{N}$ and charmed mesons, i.e., $\left\langle e_{u}\right\rangle=-\frac{2}{3}$ ) and from $\langle P|r| S\rangle=2.367 \mathrm{GeV}$ in Ref. [79], we obtain $\kappa_{E 1}^{\prime}=2.6$.

In the second case, we use the electromagnetic decays of the $D_{1(2)}^{(*)}$ charmed mesons, which are described by the nonrelativistic amplitude

$$
\mathcal{A}\left(D_{1(2)}^{(*)} \rightarrow D^{(*)} \gamma\right)=\left\langle e_{q}\right\rangle d_{E}^{\prime} \vec{\Sigma}_{L} \cdot\left(\partial_{0} \vec{A}-\vec{\partial} A_{0}\right),
$$

from which the $D_{2}^{* 0} \rightarrow D^{* 0} \gamma$ decay reads

$$
\Gamma\left(D_{2}^{* 0} \rightarrow D^{* 0} \gamma\right)=\frac{4 \alpha}{3} \frac{m\left(D^{* 0}\right)}{m\left(D_{2}^{* 0}\right)} q^{3}\left|d_{E}^{\prime}\right|^{2},
$$

with $\alpha$ the fine structure constant and $q$ the momentum of the photon. If we use this decay width as calculated in Ref. [78] $(\Gamma=895 \mathrm{keV}, q=410 \mathrm{MeV})$, then we will arrive at $\kappa_{E 1}^{\prime}=3.6$.

For the magnetic quadrupolar moment $\left(Q_{M}^{q}\right)$, it can be obtained from the matrix elements of the M2 operator [108],

$\hat{Q}_{M}^{q}=\frac{e_{q}}{2 m_{q}}\left[\frac{1}{2}\left(\sigma_{q i} r_{j}+\sigma_{q j} r_{i}\right)+\frac{2}{3}\left(l_{q i} r_{j}+l_{q j} r_{i}\right)\right]$,

with $\mu_{q}, \vec{\sigma}_{q}$ and $\vec{l}_{q}$ as defined below Eq. (A1). The matrix element of this operator will be proportional to $Q_{M}^{q}$,

$$
\left\langle D_{1(2)}^{(*)}\left|\hat{Q}_{M}^{q}\right| D^{(*)}\right\rangle=Q_{M}^{q} Q_{L i j},
$$

from which we obtain

$$
Q_{M}^{q}=-\frac{e_{q}}{2 m_{q}} \frac{\langle P|r| S\rangle}{\sqrt{3}}=\frac{e_{q}}{2 m_{q}} d_{E}^{\prime} .
$$

If we use the quark-model calculations of Ref. [79], where $m_{u}=0.22 \mathrm{GeV}$ and $\langle P|r| S\rangle=2.367 \mathrm{GeV}$, we arrive to $\kappa_{M 2}^{\prime}=10.3$. Instead, if we determine $d_{E}^{\prime}$ from Ref. [78] (where $m_{u}=0.33 \mathrm{GeV}$ ) as below Eq. (A14), we obtain $\kappa_{M 2}^{\prime}=11.1$. In this case, these two determinations yield similar results (with the average being $\kappa_{M 2}^{\prime}=10.7$ ), but this agreement is probably fortuitous, and the uncertainties should be of the same relative size as for $\kappa_{E 1}^{\prime}$. Indeed, if we simply rewrite $\kappa_{M 2}^{\prime}=\left(m_{N} / m_{u}\right) \kappa_{E 1}^{\prime}$ and vary $m_{u}$ independently of $\kappa_{E 1}^{\prime}$, we will obtain instead the $\kappa_{M 2}^{\prime}=7.4-15.4$ window, which is more in line with what to expect from $\kappa_{E 1}^{\prime}$.

\section{APPENDIX B: DIFFICULTIES WITH THE LIGHT-MESON EXCHANGE MODEL}

Here we consider a few theoretical difficulties with the light-meson exchange picture. The first is the nature of the scalar meson, which is not a pure $q \bar{q}$ state and contains tetraquark and molecular components as well (e.g., the $\sigma$ appears as a wide resonance in $\pi \pi$ scattering, check the recent review [109] and references therein). The part of the $\sigma$ which is expected to manifest at the scale we are saturating the couplings (i.e., $\Lambda \sim m_{V}$ ) is the $q \bar{q}$, with the tetraquark components playing a more important role at longer distances and manifesting themselves as the twopion exchange potential. Dealing with this issue actually requires to also consider the large width of the $\sigma$ (check the discussion below), yet the previous observation suggests treating the $\sigma$ that appears in the meson exchange model as a standard meson, though with properties that might differ from the physical $\sigma$ (a point of view which is, for instance, followed in the meson theory of nuclear forces $[32,33]$ ).

The second is the width of the scalar meson, which raises the issue of how this affects its exchange potential. Several solutions exist in the literature, of which we underline the following three: (i) to treat the exchanged $\sigma$ as a narrow effective degree of freedom, where its mass and coupling within light-meson exchange models are not necessarily the ones corresponding to a physical $\sigma[32,33]$, (ii) the twopole approximation of Binstock and Bryan [110], in which the integral of the $\sigma$ propagator over its mass distribution is approximated as the sum of two narrow particles, one lighter and one heavier than the physical $\sigma$, and (iii) the treatment by Flambaum and Shuryak [111], in which the previous integral is approximated as the sum of several contributions, of which the two most important ones are the one corresponding to the $\sigma$ pole (equivalent to the exchange of a narrow $\sigma$, but with a weaker coupling) and another corresponding to the exchange of its decay products (twopion exchange). Here we choose the effective $\sigma$ solution, which is the simplest and the one originally adopted in the meson theory of nuclear forces. Yet, we notice that the RG equation, as applied to saturation, actually relates these three solutions, as a decrease in the mass of the sigma increases its effective strength, while the presence of 
medium range two-pion exchange effects can be in turn substituted by a stronger sigma exchange. Indeed this is what actually happens in the meson exchange theory of the nuclear force, where models in which there is no two-pion exchange require a stronger $\sigma$ coupling $[32,33]$ than models which include it [112].

A third problem is the exchange of heavier light mesons with the same quantum numbers as the scalar and vector mesons (e.g., the $\sigma$ can mix with the $f_{0}(1370)$, scalar glueballs, and other $0^{++}$mesons). Again, RG-improved saturation indicates that the heavier light mesons can be included via the formula

$$
C^{\mathrm{sat}}\left(m_{V}\right)=\sum_{M} f_{\text {sup }}\left(\frac{m_{V}}{m_{M}}\right) C^{M}\left(m_{M}\right),
$$

where $M$ and $m_{M}$ denote a given meson and its mass, and $f_{\text {sup }}$ is a suppression (or, if $m_{M}<m_{V}$, enhancement) factor. For mesons with a mass similar to the saturation scale (i.e., $\Lambda \sim m_{V}$ ), the suppression factor is expected to be $f_{\text {sup }}(x)=x^{\alpha}$ with $\alpha=1$, as previously explained below Eq. (11). However, if the mass is dissimilar, then this suppression factor should deviate more and more from the previous ansatz. Besides, $f_{\text {sup }}(x)=x^{\alpha}$ is only valid for light mesons with a mass not too different from the vector mesons and eventually the finite size of the hadrons has to be taken into account, which will probably lead to a considerably larger suppression factor. The previous discussion suggests nonetheless that heavier light mesons can actually be accounted for by a redefinition of the effective couplings of the $\sigma, \rho$, and $\omega$ mesons, though the modifications are expected to be small owing to the aforementioned suppression of heavier meson contributions. Owing to the phenomenological nature of the relations we have used to obtain the couplings and the aforementioned suppression, we consider that this redefinition is not necessary.

\section{APPENDIX C: PION EXCHANGE EFFECTS}

Here we revisit the assumption that pion exchanges are a perturbative effect for the two-hadron systems we are considering. For this we will explicitly include the onepion exchange (OPE) potential in a few selected molecules and calculate the binding energy shift $\Delta B_{\mathrm{mol}}^{\mathrm{OPE}}$ that it entails. As we will see, $\Delta B_{\mathrm{mol}}^{\mathrm{OPE}}$ lies, in general, within the binding uncertainties we have previously calculated.

For including the OPE potential, we will do as follows: as we are limiting ourselves to the $S$-wave approximation, we will only consider the spin-spin component of OPE, which is given by

$$
V_{\mathrm{OPE}}(\vec{q})=\zeta \hat{T}_{12} \hat{C}_{L 12} \frac{g_{1} g_{2}}{6 f_{\pi}^{2}} \frac{\mu_{\pi}^{2}}{\mu_{\pi}^{2}+\vec{q}^{2}}+\ldots
$$

with $\mu_{\pi}$ the effective pion mass, i.e., $\mu_{\pi}^{2}=m_{\pi}^{2}-\Delta^{2}$, where $m_{\pi} \simeq 138 \mathrm{MeV}$ is the pion mass in the isospin symmetric limit, and $\Delta$ is the mass difference between the hadrons emitting (or absorbing) the virtual pion in each of the vertices, in case they are different (e.g., the $D^{*} \bar{D} \rightarrow D \bar{D}^{*}$ case, check, for instance, Ref. [20] for a more detailed discussion); the dots have the same meaning as in Eq. (6), and $\hat{T}_{12}, \hat{C}_{L 12}$ were already defined below Eq. (12). We project this potential into $S$ waves, yielding

$$
\left\langle p^{\prime}\left|V_{\mathrm{OPE}}\right| p\right\rangle=\zeta \hat{T}_{12} \hat{C}_{L 12} \frac{g_{1} g_{2}}{24 f_{\pi}^{2}} \frac{\mu_{\pi}^{2}}{p p^{\prime}} \log \left[\frac{\mu_{\pi}^{2}+\left(p+p^{\prime}\right)^{2}}{\mu_{\pi}^{2}+\left(p-p^{\prime}\right)^{2}}\right] .
$$

To obtain the molecular potential, we add OPE to the contact-range potential and regularize

$$
\left\langle p^{\prime}\left|V_{\mathrm{mol}}\right| p\right\rangle=\left(C_{\mathrm{mol}}^{\mathrm{sat}}\left(\Lambda_{H}\right)+\left\langle p^{\prime}\left|V_{\mathrm{OPE}}\right| p\right\rangle\right) f\left(\frac{p^{\prime}}{\Lambda_{H}}\right) f\left(\frac{p}{\Lambda_{H}}\right),
$$

where $f(x)$ is the regulator function (specifically, the Gaussian regulator $\left.f(x)=e^{-x^{2}}\right)$. This potential is plugged into the bound state equation

$$
\phi(k)+2 \mu_{\mathrm{mol}} \int_{0}^{\infty} \frac{p^{2} d p}{2 \pi^{2}} \frac{\left\langle k\left|V_{\mathrm{mol}}\right| p\right\rangle}{p^{2}+\gamma_{\mathrm{mol}}^{2}} \phi(p)=0,
$$

where, contrary to the purely contact-range case, the previous equation cannot be solved analytically or semianalytically when OPE is included. The solution is obtained numerically instead by discretizing the bound state equation, after which it becomes a linear system that can be solved by standard means, where $\gamma_{\text {mol }}$ is calculated by finding the zeros of the determinant of the matrix representing the linear system.

If we now define

$$
\Delta B_{\mathrm{mol}}^{\mathrm{OPE}}=B_{\mathrm{mol}}^{\mathrm{OPE}}-B_{\mathrm{mol}},
$$

for the $D^{*} \bar{D}$ and $D^{*} \bar{D}^{*}$ systems $\left(g_{1}=g_{2}=0.6\right)$, then we obtain

$$
\begin{array}{cc}
\Delta B_{\mathrm{mol}}^{\mathrm{OPE}} \approx+0.0 \mathrm{MeV} & \text { for } 1^{++} D^{*} \bar{D}, \\
\Delta B_{\mathrm{mol}}^{\mathrm{OPE}}=-1.8 \mathrm{MeV} & \text { for } 2^{++} D^{*} \bar{D}^{*},
\end{array}
$$

which lies within the uncertainties we already have and where for the $D^{*} \bar{D}$ system we have approximated the effective pion mass to zero as $m\left(D^{*}\right)-m(D) \approx m_{\pi}$. For the $\Sigma_{c} \bar{D}^{*}$ and $\Sigma_{c}^{*} \bar{D}^{*}$ systems $\left(g_{1}=0.84, g_{2}=0.6\right)$, we obtain

$$
\Delta B_{\mathrm{mol}}^{\mathrm{OPE}}=-1.6 \mathrm{MeV} \text { for } \frac{1}{2}^{-} \bar{D}^{*} \Sigma_{c},
$$




$$
\begin{array}{cc}
\Delta B_{\mathrm{mol}}^{\mathrm{OPE}}=+1.6 \mathrm{MeV} & \text { for } \frac{3^{-}}{2} \bar{D}^{*} \Sigma_{c}, \\
\Delta B_{\mathrm{mol}}^{\mathrm{OPE}}=-1.3 \mathrm{MeV} & \text { for } \quad \frac{1^{-}}{2} \bar{D}^{*} \Sigma_{c}^{*}, \\
\Delta B_{\mathrm{mol}}^{\mathrm{OPE}}=-1.2 \mathrm{MeV} & \text { for } \frac{3^{-}}{2} \bar{D}^{*} \Sigma_{c}^{*}, \\
\Delta B_{\mathrm{mol}}^{\mathrm{OPE}}=+2.5 \mathrm{MeV} & \text { for } \frac{5^{-}}{2} \bar{D}^{*} \Sigma_{c}^{*},
\end{array}
$$

which again lies within the estimated uncertainties of the model. Finally, for the $N N$ system $\left(g_{1}=g_{2}=1.29\right)$, the effect of OPE happens to be larger,

$$
\begin{array}{r}
\Delta B_{\mathrm{mol}}^{\mathrm{OPE}}=+1.8 \mathrm{MeV} \text { for the }{ }^{1} S_{0} \text { channel, } \\
\Delta B_{\mathrm{mol}}^{\mathrm{OPE}}=+2.5 \mathrm{MeV} \text { for the deuteron, }
\end{array}
$$

which is about twice the size of the uncertainties we previously estimated for these two systems. This suggests that the present model could be improved in the light baryon sector by explicitly including OPE in the future.

\section{APPENDIX D: COUPLED CHANNEL EFFECTS}

Here we consider a few examples of how coupled channel effects might affect the predictions we have made. The selected systems are (i) $\Sigma_{c} \bar{D}^{*}-\Sigma_{c}^{*} \bar{D}^{*}$, (ii) $\Xi_{c}^{\prime} \bar{D}-\Xi_{c} \bar{D}^{*}$ and $\Xi_{c} \bar{D}^{*}-\Xi_{c}^{*} \bar{D}$, (iii) $D \bar{D}-D_{s} \bar{D}_{s}$, and (iv) $D^{*} \bar{D}_{1}-D^{*} \bar{D}_{1}^{*}$. In general, these effects are smaller than the uncertainties we have already estimated. Yet, there might be exceptions in which coupled channel effects could play an important role.

\section{The $\boldsymbol{\Sigma}_{c} \overline{\boldsymbol{D}}^{*}-\boldsymbol{\Sigma}_{c}^{*} \overline{\boldsymbol{D}}^{*}$ states}

We begin with the $P_{c}(4440)$ and $P_{c}(4457)$ pentaquark states, which in our molecular model are $J=\frac{3}{2}$ and $\frac{1}{2} \Sigma_{c} \bar{D}^{*}$ bound states. It happens that the $\Sigma_{c} \bar{D}^{*}$ and $\Sigma_{c}^{*} \bar{D}^{*}$ thresholds are close, where the mass gap is $64.6 \mathrm{MeV}$, and thus it might be sensible to explicitly check whether this coupled channel effect could play a significant role in the description of the $P_{c}(4440 / 4457)$. The mechanism by which the two channels mix is the M1 interaction term, i.e., $C_{1} \hat{\vec{S}}_{L 1}$. $\hat{\vec{S}}_{L 2}$ in Eq. (1). The evaluation of the spin-spin operator for $\Sigma_{c} \bar{D}^{*}-\Sigma_{c}^{*} \bar{D}^{*}$ yields

$$
\begin{aligned}
& \hat{\vec{S}}_{L 1} \cdot \hat{\vec{S}}_{L 2}=\left(\begin{array}{cc}
-\frac{4}{3} & -\frac{\sqrt{2}}{3} \\
-\frac{\sqrt{2}}{3} & -\frac{5}{3}
\end{array}\right) \quad \text { for } J=\frac{1}{2} \\
& \text { and }\left(\begin{array}{cc}
+\frac{2}{3} & -\frac{\sqrt{5}}{3} \\
-\frac{\sqrt{5}}{3} & -\frac{2}{3}
\end{array}\right) \quad \text { for } J=\frac{3}{2},
\end{aligned}
$$

from which we can solve the coupled channel version of the bound state equation, resulting in

$$
\begin{array}{r}
M\left(\Sigma_{c} \bar{D}^{*}, \frac{1}{2}\right)=4459.6(4458.9) \mathrm{MeV}, \\
M\left(\Sigma_{c}^{*} \bar{D}^{*}, \frac{1}{2}\right)=4525.5-i 0.1(4525.4) \mathrm{MeV}, \\
M\left(\Sigma_{c} \bar{D}^{*}, \frac{3}{2}\right)=4444.3(4445.2) \mathrm{MeV}, \\
M\left(\Sigma_{c}^{*} \bar{D}^{*}, \frac{3}{2}\right)=4520.7-i 0.4(4520.2) \mathrm{MeV} .
\end{array}
$$

These masses are close to the central value of the single channel calculation (i.e., the values in parentheses), from which we are driven to the conclusion that coupled channel effects are small in the pentaquark case.

\section{The $\boldsymbol{\Xi}_{c}^{\prime} \overline{\boldsymbol{D}}-\boldsymbol{\Xi}_{c} \overline{\boldsymbol{D}}^{*}$ and $\boldsymbol{\Xi}_{c} \overline{\boldsymbol{D}}^{*}-\boldsymbol{\Xi}_{c}^{*} \overline{\boldsymbol{D}}$ states}

A second example is the $P_{c s}(4459)$ pentaquark, which in the single channel approximation is considered a $\Xi_{c} \bar{D}^{*}$ bound state. Owing to the strange content of the $\Xi_{c}$ charmed baryon, it is not clear what its coupling to the scalar meson is. On the one hand, the naive expectation is that the $\sigma$ does not contain a large $s \bar{s}$ component (if we assume it to be a $q \bar{q}$ state, which is not clear to begin with). If we combine this observation with the OZI (OkuboZweig-Iizuka) rule, the coupling of the $\sigma$ to the strange quarks within a baryon should be smaller than to the $u$ and $d$ quarks. On the other hand, the OZI rule is known to fail in the $0^{++}$sector [113-116], which implies that the $\sigma$ should not be constrained by it. From this we might expect a similar coupling for all the $u, d$, and $s$ light quarks. We will adopt this second view, which implies $g_{S}=6.8$ for the $\Xi_{c}$. With this choice we obtain degenerate $J=\frac{1}{2}, \frac{3}{2}$ bound states with a mass of

$$
M\left(\Xi_{c} \bar{D}^{*}\right)=4466.9 \mathrm{MeV},
$$

which is to be compared with the experimental mass $M\left(P_{c s}\right)=4458.8 \pm 2.0_{-1.1}^{+4.7} \mathrm{MeV}$ [52].

However, there are two nearby thresholds to be taken into account, the $\Xi_{c}^{\prime} \bar{D}$ and $\Xi_{c}^{*} \bar{D}$ for the $J=\frac{1}{2}$ and $\frac{3}{2}$ cases, respectively. The $\Xi_{c} \rightarrow \Xi_{c}^{(\prime / *)}$ transition can be described by the Lagrangian

$$
\mathcal{L}_{M 1}=\frac{f_{V}}{2 M} d_{L 0}^{\dagger} \epsilon_{i j k} \epsilon_{L i} \partial_{j} V_{k} d_{L 1}+\text { C.C. },
$$

where $d_{L 0}$ and $d_{L 1}$ are fields representing the $S=0$ and 1 light diquarks within the $\Xi_{c}$ and $\Xi_{c}^{\prime} / \Xi_{c}^{*}$ baryons, respectively, and $\vec{\epsilon}_{L}$ is the polarization vector of the $S=1$ light diquark. This Lagrangian can be matched to the electromagnetic one, 


$$
\mathcal{L}_{M 1}=\mu(1 \rightarrow 0) d_{L 0}^{\dagger} \epsilon_{i j k} \epsilon_{L i} \partial_{j} A_{k} d_{L 1}+\text { C.C., }
$$

with the diquark transition magnetic moment given by $\mu(1 \rightarrow 0)=\mu\left(q_{1}\right)-\mu\left(q_{2}\right)$, with $q_{1}=u, d$ and $q_{2}=s$ in the case at hand. The actual $\Xi_{c}^{\prime} \bar{D}-\Xi_{c} \bar{D}^{*}$ and $\Xi_{c} \bar{D}^{*}-\Xi_{c}^{*} \bar{D}$ transitions only involve the $\rho$ and $\omega$ vector mesons, and thus we can ignore the contribution from the strange meson to the magnetic moment when applying vectormeson dominance. This leads to $\kappa_{V}=2.9$ for the transition. The saturation of the coupling for the transition yields

$$
C^{\mathrm{sat}}=g_{V 1} g_{V 2}\left[\zeta+\hat{T}_{12}\right] \frac{\kappa_{V 1} \kappa_{V 2}}{6 M^{2}} \vec{\epsilon}_{L 1} \cdot \vec{\sigma}_{L 2}
$$

where the index $i=1,2$ represents the $\Xi_{c} / \Xi_{c}^{\prime} / \Xi_{c}^{*}$ charmed baryons and $\bar{D}^{(*)}$ charmed antimeson, respectively. The evaluation of the light spin-spin operator yields $\mid \vec{\epsilon}_{L 1}$. $\vec{\sigma}_{L 2} \mid=1$ for both $J=\frac{1}{2}$ and $\frac{3}{2}$. From this, we solve the coupled channel bound state equation and obtain

$$
\begin{gathered}
M\left(\Xi_{c} \bar{D}^{*}, J=\frac{1}{2}\right)=4468.4-i 1.4(4466.9) \mathrm{MeV}, \\
M\left(\Xi_{c} \bar{D}^{*}, J=\frac{3}{2}\right)=4464.4(4466.9) \mathrm{MeV},
\end{gathered}
$$

where the value in parentheses is the previous single channel calculation. In this latter case, the $J=\frac{3}{2}$ state is close to the experimental single peak solution, but it also compares well with the two peak solution considered in Ref. [52], $M_{1}=4454.9 \pm 2.7 \mathrm{MeV}$ and $M_{2}=4467.8 \pm 3.7 \mathrm{MeV}$, suggesting that the spin of the lower (higher) mass state should be $J=\frac{3}{2}\left(\frac{1}{2}\right)$.

\section{The $D \bar{D}-D_{s} \bar{D}_{s}$ states}

A second example is the $D \bar{D}$ system, for which a bound state has been predicted in the lattice [117] with $B_{\text {mol }}=4.0_{-3.7}^{+5.0}$. Here we predict a virtual state instead (which could bind within uncertainties), but we did not include the $D \bar{D}-D_{s} \bar{D}_{s}$ coupled channel dynamics of Ref. [117]. Thus it is worth the effort to explore the importance of this channel.

For the coupled channel dynamics, the $D \bar{D}-D_{s} \bar{D}_{s}$ transition potential is given by vector-meson $\left(K^{*}(890)\right)$ exchange

$$
\begin{aligned}
& V\left(H \bar{H}-H_{s} \bar{H}_{s}\right) \\
& \quad=-2 \sqrt{2} \frac{g_{V}^{2}}{q^{2}+m_{K^{*}}^{2}}\left[1+\kappa_{V}^{2} \frac{m_{K^{*}}^{2}}{6 M^{2}} \hat{\vec{S}}_{L 1} \cdot \hat{\vec{S}}_{L 2}\right],
\end{aligned}
$$

with $H=D, D^{*}$ and $H_{s}=D_{s}, D_{s}^{*}$ and $V=K^{*}$, where $g_{V}$ and $\kappa_{V}$ are the standard couplings for this system, and the $2 \sqrt{2}$ factor originates from SU(3)-flavor symmetry. For the
$D_{s} \bar{D}_{s}$ diagonal potential, it will be given by scalar- and vector-meson $(\phi(1020))$ exchange

$V\left(H_{s} \bar{H}_{s}\right)=-\frac{g_{S}^{\prime 2}}{q^{2}+m_{S}^{2}}-2 \frac{g_{V}^{2}}{q^{2}+m_{\phi}^{2}}\left[1+\kappa_{V}^{2} \frac{m_{\phi}^{2}}{6 M^{2}} \hat{\vec{S}}_{L 1} \cdot \hat{\vec{S}}_{L 2}\right]$,

where $g_{S}^{\prime}$ refers to the coupling of the $\sigma$ to the $D_{s}^{(*)}$ meson, and $m_{\phi}=1020 \mathrm{MeV}$ is the mass of the $\phi(1020)$. Regarding $g_{S}^{\prime}$ and the $D_{s}^{(*)}$, we follow the same line of argumentation as for the $\Xi_{c}$ in Appendix D 2 and take $g_{S}^{\prime}=3.4$. The saturated couplings read

$$
\begin{aligned}
C & \left(H \bar{H}-H_{s} \bar{H}_{s}\right) \\
= & -2 \sqrt{2}\left(\frac{m_{V}}{m_{K^{*}}}\right)^{\alpha} \frac{g_{V}^{2}}{m_{K^{*}}^{2}}\left[1+\kappa_{V}^{2} \frac{m_{K^{*}}^{2}}{6 M^{2}} \hat{\vec{S}}_{L 1} \cdot \hat{\vec{S}}_{L 2}\right], \quad(\mathrm{D} 15) \\
C & \left(H_{S} \bar{H}_{s}\right) \\
= & -\left(\frac{m_{V}}{m_{S}}\right)^{\alpha} \frac{g_{S}^{\prime 2}}{m_{S}^{2}}-2\left(\frac{m_{V}}{m_{\phi}}\right)^{\alpha} \frac{g_{V}^{2}}{m_{\phi}^{2}}\left[1+\kappa_{V}^{2} \frac{m_{\phi}^{2}}{6 M^{2}} \hat{\vec{S}}_{L 1} \cdot \hat{\vec{S}}_{L 2}\right],
\end{aligned}
$$

and concrete calculations yield

$$
\begin{gathered}
M(D \bar{D})=3733.5^{V}\left(3733.0^{V}\right) \mathrm{MeV}, \\
M\left(D_{s} \bar{D}_{s}\right)=3920.9^{V}-i 1.7^{V}\left(3921.7^{V}\right) \mathrm{MeV}
\end{gathered}
$$

where the superscript $V$ indicates a virtual state solution, and the masses in parentheses represent the prediction of the single channel calculation. That is, this coupled channel effect indeed provides an attractive contribution to the $D \bar{D}$ system (about half a MeV), but it is still small in comparison with the uncertainties we have for the location of the $D \bar{D}$.

\section{The $D^{*} \bar{D}_{1}-D^{*} \bar{D}_{1}^{*}$ states}

The mass difference between the $D_{1}\left(S_{L}=\frac{3}{2}\right)$ and $D_{1}^{*}$ $\left(S_{L}=\frac{1}{2}\right)$ charmed mesons is small,

$$
m\left(D_{1}\right)-m\left(D_{1}^{*}\right)=(10 \pm 9) \mathrm{MeV},
$$

where most of the uncertainty comes from the broad $D_{1}^{*}$ charmed meson. As a consequence, if we try to explain the $Y(4360)$ as a $D^{*} \bar{D}_{1}$ molecule, it will be difficult not to consider too the possible mixing with the $D^{*} \bar{D}_{1}^{*}$ system. Indeed, if both the $Y(4260)$ and $Y(4360)$ were to be $D \bar{D}_{1}$ and $D^{*} \bar{D}_{1}$ molecules, respectively, the possible mixing of the $Y(4360)$ with the $D^{*} \bar{D}_{1}^{*}$ channel might very well explain why it is considerably broader than the $Y(4260)$. 
In the following lines we will explain how to include the $D^{*} \bar{D}_{1}-D^{*} \bar{D}_{1}^{*}$ coupled channel dynamics.

We begin with the M1 $D_{1} \rightarrow D_{1}^{*}$ vector-meson transitions, for which the Lagrangian reads

$$
\mathcal{L}_{M 1}=\frac{f_{V}}{2 M} q_{L}^{\prime \prime \dagger} \epsilon_{i j k} \Sigma_{L i} \partial_{j} V_{k} q_{L}^{\prime}+\text { C.C. }
$$

where $q_{L}^{\prime}$ and $q_{L}^{\prime \prime}$ refer to the light-quark subfield for the $S_{L}=\frac{3}{2}$ and $S_{L}=\frac{1}{2} P$-wave charmed mesons, respectively. By matching with the electromagnetic Lagrangian,

$$
\mathcal{L}_{M 1}=\mu_{q}\left(\frac{3}{2} \rightarrow \frac{1}{2}\right) q_{L}^{\prime \prime \dagger} \epsilon_{i j k} \Sigma_{L i} \partial_{j} A_{k} q_{L}^{\prime}+\text { C.C. }
$$

we obtain $\kappa_{V}=\frac{3}{2} \mu_{u}\left(\frac{3}{2} \rightarrow \frac{1}{2}\right)$, where the transition magnetic moment can be extracted from the matrix elements of the magnetic moment operator in Eq. (A1), yielding $\mu_{u}\left(\frac{3}{2} \rightarrow \frac{1}{2}\right)=\mu_{u} / \sqrt{3}$. The next is the E1 $D \rightarrow D_{1}^{*}$ vectormeson transition, which can be obtained from the matrix elements of

$$
\left\langle D_{0(1)}^{(*)}\left|\hat{d}_{E}^{q}\right| D^{(*)}\right\rangle=\left\langle e_{q}\right\rangle d_{E}^{\prime \prime} \vec{\sigma}_{L},
$$

with $d_{E}^{\prime \prime}=-\langle P|r| S\rangle / 3$, i.e., $1 / \sqrt{3}$ smaller than for the $D \rightarrow$ $D_{1}$ family of transitions. This gives us $\kappa_{E 1}^{\prime \prime}=\kappa_{E 1}^{\prime} / \sqrt{3}$.

The saturated coupling for the $D^{*} \bar{D}_{1}-D^{*} \bar{D}_{1}^{*}$ transition reads

$$
C^{\text {sat }} \propto C_{M 1}\left(\vec{\sigma}_{L 1} \cdot \vec{\Sigma}_{L 2}\right)_{D}+C_{E 1}\left(\vec{\sigma}_{L 1} \cdot \vec{\Sigma}_{L 2}\right)_{E}
$$

where the $D$ and $E$ subscripts stand for "direct" $\left(D^{*} \bar{D}_{1} \rightarrow D^{*} \bar{D}_{1}^{*}\right)$ and "exchange" $\left(D^{*} \bar{D}_{1} \rightarrow D_{1}^{*} \bar{D}^{*}\right)$ terms, the difference being that the sign of the exchange term depends on the $C$-parity of the system under consideration (for $J^{P C}=1^{--}$the exchange and direct terms have the same sign). The $C_{M 1}$ and $C_{E 1}$ contributions read

$$
\begin{gathered}
C_{M 1}=+g_{V}^{2}\left[\zeta+\hat{T}_{12}\right] \frac{\kappa_{M 1} \kappa_{M 1}^{\prime}}{6 M^{2}} \\
C_{E 1}=-g_{V}^{2}\left[\zeta+\hat{T}_{12}\right] \frac{\kappa_{E 1}^{\prime} \kappa_{E 1}^{\prime \prime}}{4 M^{2}}\left(\frac{m_{V}}{\mu_{V}}\right)^{\alpha}\left(\frac{\omega^{2}+\frac{1}{3} \mu_{V}^{2}}{\mu_{V}^{2}}\right),
\end{gathered}
$$

where for $J^{P C}=1^{--}$the $\mathrm{M} 1$ and E1 terms end up interfering destructively, leading to relatively weak coupled channel dynamics (despite the two thresholds being so close). If we use $M=m_{N}, \kappa_{M 1}=\sqrt{3} \kappa_{M 1}{ }^{\prime}=2.9$ and $\kappa_{E 1}^{\prime}=\sqrt{3} \kappa_{E 1}^{\prime \prime} \simeq 3.1$, then we end up with a weakly bound $D^{*} D_{1}-D^{*} D_{1}^{*}$ state with a mass of $4420 \pm 9 \mathrm{MeV}$. However, if we employ $\kappa_{E 1}^{\prime}=\sqrt{3} \kappa_{E 1}^{\prime \prime} \simeq 3.9$ [the value of the E1 coupling that reproduces the $Y(4260)]$ and the higher end value of the M2 coupling for this choice of $\kappa_{E 1}^{\prime}$, i.e., $\kappa_{M 2}^{\prime} \simeq 16.7$, then we will predict a bound state with mass of $4417_{-8}^{+6} \mathrm{MeV}$.
[1] Y. Ne'eman, Nucl. Phys. 26, 222 (1961).

[2] M. Gell-Mann, Phys. Rev. 125, 1067 (1962).

[3] N. Isgur and G. Karl, Phys. Rev. D 18, 4187 (1978).

[4] N. Isgur and G. Karl Phys. Rev. D 19, 2653 (1979); 23, 817(E) (1981).

[5] N. Isgur and G. Karl, Phys. Rev. D 20, 1191 (1979).

[6] S. Godfrey and N. Isgur, Phys. Rev. D 32, 189 (1985).

[7] E. Eichten, K. Gottfried, T. Kinoshita, K. D. Lane, and T.-M. Yan, Phys. Rev. D 17, 3090 (1978); 21, 313(E) (1980).

[8] E. Eichten, K. Gottfried, T. Kinoshita, K. D. Lane, and T.-M. Yan, Phys. Rev. D 21, 203 (1980).

[9] N. Brambilla, A. Pineda, J. Soto, and A. Vairo, Nucl. Phys. B566, 275 (2000).

[10] N. Brambilla, A. Pineda, J. Soto, and A. Vairo, Rev. Mod. Phys. 77, 1423 (2005).

[11] H.-X. Chen, W. Chen, X. Liu, and S.-L. Zhu, Phys. Rep. 639, 1 (2016).

[12] A. Hosaka, T. Iijima, K. Miyabayashi, Y. Sakai, and S. Yasui, Prog. Theor. Exp. Phys. 2016, 062 C01 (2016).

[13] R. F. Lebed, R. E. Mitchell, and E. S. Swanson, Prog. Part. Nucl. Phys. 93, 143 (2017).
[14] F.-K. Guo, C. Hanhart, U.-G. Meißner, Q. Wang, Q. Zhao, and B.-S. Zou, Rev. Mod. Phys. 90, 015004 (2018).

[15] M. Voloshin and L. Okun, JETP Lett. 23, 333 (1976).

[16] A. De Rujula, H. Georgi, and S. Glashow, Phys. Rev. Lett. 38, 317 (1977).

[17] M. Karliner and J. L. Rosner, Phys. Rev. Lett. 115, 122001 (2015).

[18] X.-K. Dong, F.-K. Guo, and B.-S. Zou, Prog. Phys. 41, 65 (2021).

[19] F.-Z. Peng, M.-Z. Liu, M. S. Sánchez, and M. P. Valderrama, Phys. Rev. D 102, 114020 (2020).

[20] M. P. Valderrama, Phys. Rev. D 85, 114037 (2012).

[21] J.-X. Lu, L.-S. Geng, and M. P. Valderrama, Phys. Rev. D 99, 074026 (2019).

[22] M. P. Valderrama, Eur. Phys. J. A 56, 109 (2020).

[23] M. P. Valderrama and D. R. Phillips, Phys. Rev. Lett. 114, 082502 (2015).

[24] M. P. Valderrama, Int. J. Mod. Phys. E 25, 1641007 (2016).

[25] R. E. Langer, Phys. Rev. 51, 669 (1937).

[26] J. J. Sakurai, Ann. Phys. (N.Y.) 11, 1 (1960).

[27] K. Kawarabayashi and M. Suzuki, Phys. Rev. Lett. 16, 255 (1966). 
[28] Riazuddin and Fayyazuddin, Phys. Rev. 147, 1071 (1966).

[29] D. O. Riska and G. E. Brown, Nucl. Phys. A679, 577 (2001).

[30] M. Gell-Mann and M. Levy, Nuovo Cimento 16, 705 (1960).

[31] P. Zyla et al. (Particle Data Group), Prog. Theor. Exp. Phys. 2020, 083C01 (2020).

[32] R. Machleidt, K. Holinde, and C. Elster, Phys. Rep. 149, 1 (1987).

[33] R. Machleidt, Advances in Nuclear Physics (Springer, Boston, 1989).

[34] R. Aaij et al. (LHCb Collaboration), Phys. Rev. Lett. 122, 222001 (2019).

[35] M.-Z. Liu, Y.-W. Pan, F.-Z. Peng, M. S. Sánchez, L.-S. Geng, A. Hosaka, and M. P. Valderrama, Phys. Rev. Lett. 122, 242001 (2019).

[36] M. P. Valderrama, Phys. Rev. D 100, 094028 (2019).

[37] C. Xiao, J. Nieves, and E. Oset, Phys. Rev. D 100, 014021 (2019).

[38] M.-L. Du, V. Baru, F.-K. Guo, C. Hanhart, U.-G. Meißner, J. A. Oller, and Q. Wang, Phys. Rev. Lett. 124, 072001 (2020).

[39] M.-Z. Liu, T.-W. Wu, M. S. Sánchez, M. P. Valderrama, L.-S. Geng, and J.-J. Xie, Phys. Rev. D 103, 054004 (2021).

[40] M.-Z. Liu, T.-W. Wu, M. P. Valderrama, J.-J. Xie, and L.-S. Geng, Phys. Rev. D 99, 094018 (2019).

[41] N. A. Tornqvist, Z. Phys. C 61, 525 (1994).

[42] J. Nieves and M. P. Valderrama, Phys. Rev. D 86, 056004 (2012).

[43] D. Gamermann, E. Oset, D. Strottman, and M. V. Vacas, Phys. Rev. D 76, 074016 (2007).

[44] C. Xiao and E. Oset, Eur. Phys. J. A 49, 52 (2013).

[45] E. S. Swanson, Phys. Lett. B 598, 197 (2004).

[46] Y. Dong, A. Faessler, T. Gutsche, and V. E. Lyubovitskij, J. Phys. G 38, 015001 (2011).

[47] F.-K. Guo, C. Hanhart, Y. S. Kalashnikova, U.-G. Meißner, and A. V. Nefediev, Phys. Lett. B 742, 394 (2015).

[48] A. Esposito, E. G. Ferreiro, A. Pilloni, A. D. Polosa, and C. A. Salgado, Eur. Phys. J. C 81, 669 (2021).

[49] E. Braaten, L.-P. He, K. Ingles, and J. Jiang, Phys. Rev. D 103, L071901 (2021).

[50] A. Esposito, L. Maiani, A. Pilloni, A. D. Polosa, and V. Riquer, arXiv:2108.11413.

[51] M. Ablikim et al. (BESIII Collaboration), Phys. Rev. Lett. 126, 102001 (2021).

[52] R. Aaij et al. (LHCb Collaboration), Sci. Bull. 66, 1391 (2021).

[53] Z. Yang, X. Cao, F.-K. Guo, J. Nieves, and M. P. Valderrama, Phys. Rev. D 103, 074029 (2021).

[54] L. Meng, B. Wang, and S.-L. Zhu, Phys. Rev. D 102, 111502 (2020).

[55] Z.-F. Sun and C.-W. Xiao, arXiv:2011.09404.

[56] N. Ikeno, R. Molina, and E. Oset, Phys. Lett. B 814, 136120 (2021).

[57] M. Albaladejo, F.-K. Guo, C. Hidalgo-Duque, and J. Nieves, Phys. Lett. B 755, 337 (2016).

[58] M.-J. Yan, F.-Z. Peng, M. S. Sánchez, and M. P. Valderrama, arXiv:2102.13058.
[59] H.-X. Chen, W. Chen, X. Liu, and X.-H. Liu, Eur. Phys. J. C 81, 409 (2021).

[60] F.-Z. Peng, M.-J. Yan, M. S. Sánchez, and M. P. Valderrama, Eur. Phys. J. C 81, 666 (2021).

[61] R. Chen, Phys. Rev. D 103, 054007 (2021).

[62] M.-Z. Liu, Y.-W. Pan, and L.-S. Geng, Phys. Rev. D 103, 034003 (2021).

[63] K. Tanida et al. (Belle Collaboration), in Proceedings of the 18th International Conference on Hadron Spectroscopy and Structure (World Scientific, Singapore, 2020), pp. 183-187, 10.1142/9789811219313_0028.

[64] M. Ablikim et al. (BESIII Collaboration), Phys. Rev. D 102, 031101 (2020).

[65] B. Aubert et al. (BABAR Collaboration), Phys. Rev. Lett. 95, 142001 (2005).

[66] X. Liu, X.-Q. Zeng, and X.-Q. Li, Phys. Rev. D 72, 054023 (2005).

[67] G.-J. Ding, Phys. Rev. D 79, 014001 (2009).

[68] Q. Wang, C. Hanhart, and Q. Zhao, Phys. Rev. Lett. 111, 132003 (2013).

[69] Q. Wang, M. Cleven, F.-K. Guo, C. Hanhart, U.-G. Meißner, X.-G. Wu, and Q. Zhao, Phys. Rev. D 89, 034001 (2014).

[70] M. Cleven, Q. Wang, F.-K. Guo, C. Hanhart, U.-G. Meißner, and Q. Zhao, Phys. Rev. D 90, 074039 (2014).

[71] Y.-H. Chen, L.-Y. Dai, F.-K. Guo, and B. Kubis, Phys. Rev. D 99, 074016 (2019).

[72] S.-L. Zhu, Phys. Lett. B 625, 212 (2005).

[73] F. J. Llanes-Estrada, Phys. Rev. D 72, 031503 (2005).

[74] L. Maiani, V. Riquer, F. Piccinini, and A. Polosa, Phys. Rev. D 72, 031502 (2005).

[75] S. Dubynskiy and M. Voloshin, Phys. Lett. B 666, 344 (2008).

[76] A. M. Torres, K. Khemchandani, D. Gamermann, and E. Oset, Phys. Rev. D 80, 094012 (2009).

[77] X. Li and M. B. Voloshin, Mod. Phys. Lett. A 29, 1450060 (2014).

[78] F. E. Close and E. S. Swanson, Phys. Rev. D 72, 094004 (2005).

[79] S. Godfrey, Phys. Rev. D 72, 054029 (2005).

[80] C.-W. Hwang, Eur. Phys. J. C 23, 585 (2002).

[81] D. Becirevic, E. Chang, and A. Le Yaouanc, Phys. Rev. D 80, 034504 (2009).

[82] D. Becirevic, E. Chang, L. Oliver, J.-C. Raynal, and A. Le Yaouanc, Phys. Rev. D 84, 054507 (2011).

[83] K. U. Can, G. Erkol, M. Oka, A. Ozpineci, and T. T. Takahashi, Phys. Lett. B 719, 103 (2013).

[84] K. U. Can, G. Erkol, B. Isildak, M. Oka, and T. T. Takahashi, J. High Energy Phys. 05 (2014) 125.

[85] K. U. Can, G. Erkol, B. Isildak, M. Oka, and T. T. Takahashi, Phys. Lett. B 726, 703 (2013).

[86] A. C. Cordon and E. R. Arriola, Phys. Rev. C 81, 044002 (2010).

[87] E. Wigner, Phys. Rev. 51, 106 (1937).

[88] T. Mehen, I. W. Stewart, and M. B. Wise, Phys. Rev. Lett. 83, 931 (1999).

[89] J.-W. Chen, D. Lee, and T. Schäfer, Phys. Rev. Lett. 93, 242302 (2004).

[90] A. C. Cordon and E. R. Arriola, Phys. Rev. C 78, 054002 (2008). 
[91] A. C. Cordon and E. R. Arriola, Phys. Rev. C 80, 014002 (2009).

[92] V. S. Timoteo, S. Szpigel, and E. R. Arriola, Phys. Rev. C 86, 034002 (2012).

[93] E. R. Arriola, Symmetry 8, 42 (2016).

[94] D. Lee et al., Phys. Rev. Lett. 127, 062501 (2021).

[95] F. Dyson and N. H. Xuong, Phys. Rev. Lett. 13, 815 (1964).

[96] P. Adlarson et al. (WASA-at-COSY Collaboration), Phys. Rev. Lett. 106, 242302 (2011).

[97] R. Molina, N. Ikeno, and E. Oset, arXiv:2102.05575.

[98] S. Gongyo, K. Sasaki, T. Miyamoto, S. Aoki, T. Doi, T. Hatsuda, Y. Ikeda, T. Inoue, and N. Ishii (HAL QCD Collaboration), Phys. Lett. B 811, 135935 (2020).

[99] X.-G. He, X.-Q. Li, X. Liu, and X.-Q. Zeng, Eur. Phys. J. C 51, 883 (2007).

[100] J. He, Y.-T. Ye, Z.-F. Sun, and X. Liu, Phys. Rev. D 82, 114029 (2010).

[101] J.-R. Zhang, Phys. Rev. D 89, 096006 (2014).

[102] P. G. Ortega, D. R. Entem, and F. Fernandez, Phys. Lett. B 718, 1381 (2013).

[103] B. Wang, L. Meng, and S.-L. Zhu, Phys. Rev. D 101, 094035 (2020).
[104] S. Sakai, F.-K. Guo, and B. Kubis, Phys. Lett. B 808, 135623 (2020).

[105] J. Haidenbauer, G. Krein, U.-G. Meissner, and L. Tolos, Eur. Phys. J. A 47, 18 (2011).

[106] R. Mizuk et al. (Belle Collaboration), Phys. Rev. Lett. 94, 122002 (2005).

[107] S. Acharya et al. (ALICE Collaboration), arXiv:2201.05352.

[108] R. Raab, Mol. Phys. 29, 1323 (1975).

[109] J. Pelaez, Phys. Rep. 658, 1 (2016).

[110] J. Binstock and R. Bryan, Phys. Rev. D 4, 1341 (1971).

[111] V. V. Flambaum and E. V. Shuryak, Phys. Rev. C 76, 065206 (2007).

[112] V. G. J. Stoks, R. A. M. Klomp, C. P. F. Terheggen, and J. J. de Swart, Phys. Rev. C 49, 2950 (1994).

[113] P. Geiger and N. Isgur, Phys. Rev. D 47, 5050 (1993).

[114] H. J. Lipkin and B.-s. Zou, Phys. Rev. D 53, 6693 (1996).

[115] N. Isgur and H. B. Thacker, Phys. Rev. D 64, 094507 (2001).

[116] U.-G. Meissner and J. A. Oller, Nucl. Phys. A679, 671 (2001).

[117] S. Prelovsek, S. Collins, D. Mohler, M. Padmanath, and S. Piemonte, J. High Energy Phys. 06 (2021) 035. 